\title{
REPORT
}

\section{Vps13-like proteins provide phosphatidylethanolamine for GPI anchor synthesis in the ER}

Alexandre Toulmay ${ }^{1}$, Fawn B. Whittle ${ }^{1}$, Jerry Yang ${ }^{1}$, Xiaofei Bai ${ }^{2}$, Jessica Diarra ${ }^{1}$, Subhrajit Banerjee ${ }^{1} \mathbb{D}$, Tim P. Levine ${ }^{3}$ D , Andy Golden ${ }^{2}$, and William A. Prinz ${ }^{1} \mathbb{D}$

Glycosylphosphatidylinositol (GPI) is a glycolipid membrane anchor found on surface proteins in all eukaryotes. It is synthesized in the ER membrane. Each GPI anchor requires three molecules of ethanolamine phosphate (P-Etn), which are derived from phosphatidylethanolamine (PE). We found that efficient GPI anchor synthesis in Saccharomyces cerevisiae requires Csf1; cells lacking Csf1 accumulate GPI precursors lacking P-Etn. Structure predictions suggest Csf1 is a tube-forming lipid transport protein like Vps13. Csf1 is found at contact sites between the ER and other organelles. It interacts with the ER protein Mcd4, an enzyme that adds P-Etn to nascent GPI anchors, suggesting Csf1 channels PE to Mcd4 in the ER at contact sites to support GPI anchor biosynthesis. CSF1 has orthologues in Caenorhabditis elegans (Ipd-3) and humans (KIAA1109) TWEEK); mutations in KIAA1109 cause the autosomal recessive neurodevelopmental disorder Alkuraya-Kučinskas syndrome. Knockout of Ipd-3 and knockdown of KIAA1109 reduced GPI-anchored proteins on the surface of cells, suggesting Csf1 orthologues in human cells support GPI anchor biosynthesis.

\section{Introduction}

Lipids are exchanged between cellular membranes by both vesicular and nonvesicular mechanisms. Nonvesicular trafficking requires proteins known as lipid transport proteins (LTPs). Until recently, most LTPs in eukaryotic cells were thought to transport lipids by a shuttling mechanism; the LTP extracts a lipid from one membrane, diffuses through the cytoplasm, and delivers the lipid to a second membrane (Chiapparino et al., 2016; Wong et al., 2019). In the past few years, it has been suggested that some eukaryotic LTPs use a different mechanism: they bind two membranes simultaneously and allow lipids to flow between the membranes in a hydrophobic channel formed by the LTP. This mechanism of lipid transport could be used for high-volume lipid movement (Leonzino et al., 2021; Reinisch and Prinz, 2021). Two proteins thought to use this mechanism are vacuolar protein sorting 13 (Vps13) and autophagy-related protein 2 (Atg2; Chowdhury et al., 2018; De et al., 2017; Kumar et al., 2018; Li et al., 2020; Maeda et al., 2019). Structural studies reveal that Vps13 and Atg2 have long rodlike structures with hydrophobic grooves that run from one end of the proteins to the other and could allow lipids to move between

membranes by sliding along the groove (Li et al., 2020; Valverde et al., 2019).

Both Vps13 and Atg2 are conserved from yeast to humans. Atg2 is required for the autophagosome formation (Suzuki et al., 2013). Saccharomyces cerevisiae has a single Vps13, while humans have four orthologues; all have been proposed to facilitate lipid transport at membrane contact sites (MCSs), regions where two organelles are closely apposed (Lang et al., 2015; Leonzino et al., 2021; Park et al., 2016). The four Vps13 proteins in humans are implicated in neurodegenerative disorders (Gauthier et al., 2018; Kolehmainen et al., 2003; Lesage et al., 2016; Rampoldi et al., 2001; Seong et al., 2018).

In this study, we show that $S$. cerevisiae has three proteins predicted to be structurally like Vps13 and Atg2: Csfl, Fmp27, and Hob2 (Ypr117w). The functions of Csfl, Fmp27, and Hob2 are not well understood. Fmp27 was recently shown to localize to MCSs between the ER and plasma membrane (PM) and bind phosphoinositides (Neuman et al., 2022). Cells lacking Csfl have also been shown to have defects in vesicular trafficking of proteins with glycosylphosphatidylinositol (GPI) anchors (Copic

'Laboratory of Cell and Molecular Biology, National Institute of Diabetes and Digestive and Kidney Diseases, National Institutes of Health, Bethesda, MD; 2Laboratory of Biochemistry and Genetics, National Institute of Diabetes and Digestive and Kidney Diseases, National Institutes of Health, Bethesda, MD; ${ }^{3}$ University College London, Institute of Ophthalmology, London, UK.

Correspondence to William A. Prinz: wp53m@nih.gov; Alexandre Toulmay: alexandre.toulmay@nih.gov.

(c) 2022 Toulmay et al. This article is distributed under the terms of an Attribution-Noncommercial-Share Alike-No Mirror Sites license for the first six months after the publication date (see http://www.rupress.org/terms/). After six months it is available under a Creative Commons License (Attribution-Noncommercial-Share Alike 4.0 International license, as described at https://creativecommons.org/licenses/by-nc-sa/4.0/). 
et al., 2009). GPI anchors are synthesized in the ER by a conserved multistep pathway (Fujita and Kinoshita, 2012). The anchor contains a phosphatidylinositol to which is added glycans and three ethanolamine phosphate (P-Etn) molecules. Anchor assembly and protein attachment occur on the luminal surface of the ER membrane. The P-Etn in GPI anchors is derived from phosphatidylethanolamine (PE; Menon and Stevens, 1992); thus, biosynthesis of each GPI anchor requires three PE molecules. Here, we show that Csfl is necessary for efficient P-Etn incorporation into GPI anchors.

\section{Results and discussion}

\section{Csf1, Fmp27, and Hob2 are structural homologues of Vps13}

To identify proteins that are structurally like Vps13 and Atg2 in $S$. cerevisiae, we used the remote homology detection program named HHpred, which identified Csfl, Fmp27, and Hob2. To gain more insight into the predicted structures of Csfl, Fmp27, Hob2, Vps13, and Atg2, we used the protein structure prediction algorithm RoseTTAFold (Yang et al., 2020) to generate structures of each 1,000 residues of the proteins, which we then stitched together into full-length predicted structures using the molecular visualization program PyMol. This analysis revealed the proteins form similar extended $\beta$-sheets with long hydrophobic grooves (Fig. 1 A, Video 1, Video 2, Video 3, Video 4, and Video 5). We used the structure comparison program FATCAT (Flexible structure AlignmenT by Chaining Aligned fragment pairs allowing Twists) to show the predicted structures are similar (Fig. 1, B and C). We compared the predicted structure of the first 1,000 residues of $\mathrm{Vps} 13$ and the crystal structure of $\mathrm{Vps} 13$ (Protein Data Bank accession no. 6CBC), which is of the first 335 residues (Kumar et al., 2018). The $\mathrm{P}$ value is $4.95 \mathrm{E}-11$, indicating the predicted structure aligns well with the solved structure. We also compared the full-length structures of Fmp27, Hob2, and Atg2 we generated with RoseTTAFold with the recently published predicted structures of these proteins generated by the protein structure prediction program AlphaFold (Tunyasuvunakool et al., 2021). Comparisons of the structures using FATCAT indicated they are highly similar (Fig. S1 A). Notably, FATCAT indicated that the most similar portions of the predicted structure are the extended $\beta$-sheets found in all the predicted structures, which form long hydrophobic grooves running the length of the proteins (not shown). We found that the central groove that runs the length of these proteins is lined with hydrophobic residues (Fig. S1 B), consistent with the hypothesis that lipids could slide along the grooves.

\section{Csf1, Fmp27, and Hob2 localize to multiple contact sites}

If Csfl, Fmp27, and Hob2 are LTPs that function similarly to other bridge-forming LTPs, they should localize to MCSs. A recent study indeed showed that endogenous FMP27-GFP localizes to contact sites between the ER and PM (Neuman et al., 2022). To visualize Csfl and Hob2, we initially tagged them endogenously with GFP on their C-termini, but the signal was low (not shown). Instead of using a single GFP, we endogenously tagged the proteins with seven copies of GFP11, a fragment of GFP that will self-assemble with the remainder of the protein, GFP1-10
(Makhija et al., 2021). The tagged proteins are functional (Fig. S2 A). We found that GFP-tagged Csfl, Fmp27, and Hob2 form puncta that are on or next to the ER (Fig. 2 A). Most of the puncta are at the cell cortex. The cortical puncta associate with Tcb3 (Fig. 2 B), a protein known to be at ER-PM contact sites (Toulmay and Prinz, 2012), suggesting that Csfl, Fmp27, and Hob2 localize to these contact sites. Not all Csfl, Fmp27, and Hob2 puncta are at the cell cortex; some are adjacent to mitochondria (Fig. 2 C). To determine whether some Csfl, Fmp27, and Hob2 puncta are at ER-mitochondria contact sites, we assessed whether they colocalize with the ER-mitochondria tether ERMES (ER mitochondria encounter structure). GFP-tagged Csfl, Fmp27, or Hob2 was expressed together with mCherry fused to Mmm1, one of the components of ERMES. All three Vps13-like proteins were found to be adjacent to or colocalize with $\sim 50 \%$ of Mmm1-mCherry puncta (Fig. 2, D and E), suggesting they localize to some ER-mitochondria contact sites. It is not clear why Csf1, Fmp27, or Hob2 localizes to only a subset of ERMES puncta. Together, these findings indicate that Csfl, Fmp27, or Hob2 localizes to ER-PM and some ER-mitochondria contact sites.

\section{Vps13-like proteins are dispensable for bulk lipid transport to mitochondria}

It has been suggested that tube-forming LTPs are well suited to facilitate high-volume bulk lipid transport (Leonzino et al., 2021; Reinisch and Prinz, 2021). We investigated their role in nonvesicular lipid transport to mitochondria, which is required for mitochondrial biogenesis (Petrungaro and Kornmann, 2019). We first generated a strain lacking all five Vps13-like LTPs in $S$. cerevisiae: Vps13, Atg2, Csf1, Fmp27, and Hob2. Remarkably, the strain (called $5 \Delta$ here) grew about as well as WT cells at $30^{\circ} \mathrm{C}$ on the glucose-containing media (YPD; Yeast Extract, Peptone, Dextrose and synthetic complete [SC] medium) and YPG (Yeast Extract, Peptone, Glycerol), which only allows the growth of cells with functional mitochondria (Fig. $3 \mathrm{~A}$ ). To more directly determine whether there is a defect in bulk lipid transport to mitochondria in $5 \Delta$ cells, we assessed the rate of cardiolipin (CL) synthesis. In $S$. cerevisiae, CL is produced in the mitochondrial inner membrane from phospholipids synthesized in the ER, primarily phosphatidic acid. Therefore, CL production in mitochondria requires phospholipid transport from the ER to mitochondria (Fig. 3 B; Tatsuta and Langer, 2017). To measure CL synthesis, we determined the amount of CL formed in $1 \mathrm{~h}$ by labeling cells with $\left[{ }^{3} \mathrm{H}\right]$ acetate. The amount of CL formed in $5 \Delta$ cells (expressed as a percentage of the total phospholipid synthesized) decreased $\sim 40 \%$ (Fig. $3 \mathrm{C}$ ). This suggests the five Vps13-like proteins could play a role in transporting CL precursors from the ER to mitochondria but are not required.

It is possible that the ERMES complex compensates for the lack of Vps13-like proteins in $5 \Delta$ cells. ERMES tethers mitochondria to the ER and facilitates phospholipid transport to mitochondria (Kawano et al., 2018; Kornmann et al., 2009). We generated a $5 \Delta$ strain that also lacks the ERMES complex. Because cells lacking the ERMES complex and Vps13 are not viable (Lang et al., 2015), we added an auxin-inducible degron to one of the components of ERMES, Mdm12, to allow conditional 


\section{A}

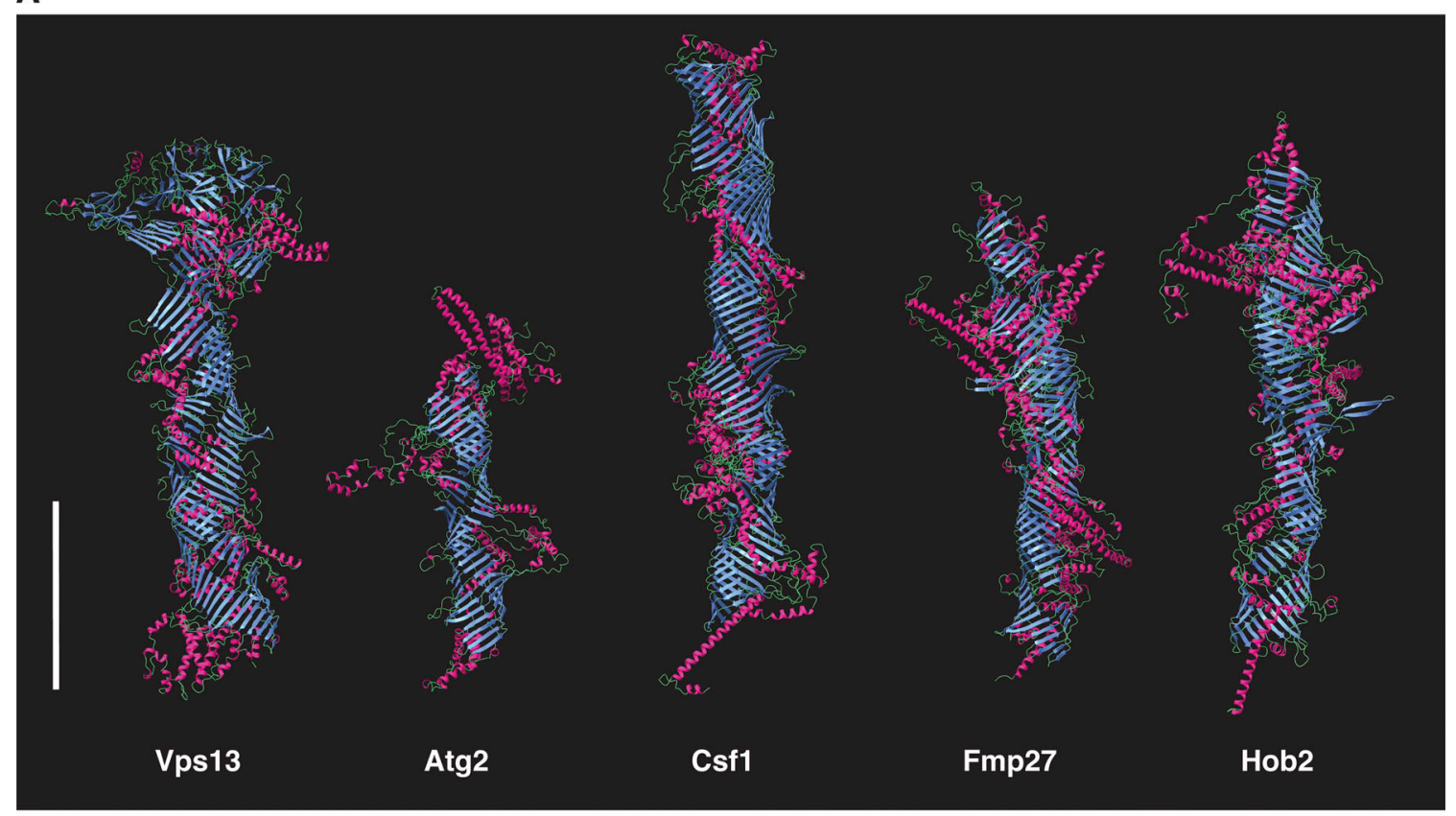

B

\begin{tabular}{|c|c|c|c|c|c|}
\hline P-values & Vps13 & Atg2 & Csf1 & Fmp27 & Hob2 \\
\hline Vps13 & $X$ & $1.89 \mathrm{E}-04$ & $3.17 \mathrm{E}-03$ & $7.69 \mathrm{E}-06$ & $4.28 \mathrm{E}-04$ \\
\hline Atg2 & $1.81 \mathrm{E}-04$ & $X$ & $8.17 \mathrm{E}-04$ & $3.95 \mathrm{E}-04$ & $2.62 \mathrm{E}-04$ \\
\hline Csf1 & $3.97 \mathrm{E}-03$ & $8.43 \mathrm{E}-04$ & $X$ & $2.42 \mathrm{E}-03$ & $1.87 \mathrm{E}-03$ \\
\hline Fmp27 & $9.16 \mathrm{E}-05$ & $3.72 \mathrm{E}-04$ & $2.42 \mathrm{E}-03$ & $X$ & $1.02 \mathrm{E}-08$ \\
\hline Hob2 & $3.81 \mathrm{E}-04$ & $2.77 \mathrm{E}-04$ & $1.69 \mathrm{E}-03$ & $9.28 \mathrm{E}-09$ & $\mathrm{X}$ \\
\hline
\end{tabular}

C

\begin{tabular}{|c|c|c|c|c|c|}
\hline P-values & Vps13 & Atg2 & Csf1 & Fmp27 & Hob2 \\
\hline Vps13 & $X$ & $1.14 \mathrm{E}-02$ & $6.04 \mathrm{E}-03$ & $1.85 \mathrm{E}-02$ & $9.00 \mathrm{E}-02$ \\
\hline Atg2 & $1.08 \mathrm{E}-02$ & $\mathrm{X}$ & $1.76 \mathrm{E}-02$ & $2.49 \mathrm{E}-02$ & $5.25 \mathrm{E}-02$ \\
\hline Csf1 & $5.45 \mathrm{E}-03$ & $2.84 \mathrm{E}-02$ & $\mathrm{X}$ & $5.09 \mathrm{E}-02$ & $6.48 \mathrm{E}-02$ \\
\hline Fmp27 & $3.38 \mathrm{E}-02$ & $2.64 \mathrm{E}-02$ & $5.21 \mathrm{E}-02$ & $X$ & $7.35 \mathrm{E}-06$ \\
\hline Hob2 & $1.03 \mathrm{E}-01$ & $5.79 \mathrm{E}-02$ & $6.40 \mathrm{E}-02$ & $4.64 \mathrm{E}-04$ & $\mathrm{X}$ \\
\hline
\end{tabular}

Figure 1. Structural similarity of Vps13, Atg2, Csf1, Fmp27, and Hob2. (A) Structure predictions generated with RoseTTAFold: $\beta$-sheets (blue), a-helices (red), and coil (green). Bar $=100 \AA$. (B and C) Significance of the structural similarity between pairs of proteins using FATCAT. P values are from pairwise comparisons of the predicted structures of the first 1,000 amino acids (B) or full-length versions (C) of the proteins. The first structure for each pairwise comparison is listed in the first column. $P$ values $>0.05$ in gray.

degradation of ERMES (Fig. S2, B-D). Elimination of any of the proteins in the ERMES complex causes it to disassemble (Kornmann et al., 2009). We found the degron causes Mdm12 to degrade within $30 \mathrm{~min}$ of adding the auxin to the medium (Fig. S2 B). Mdm12 degradation also caused mitochondria to change shape (Fig. S2 D), indicating that ERMES is not functional after Mdm12 depletion. Interestingly, we found VPS13 is the only one of the five genes encoding Vps13-like proteins that is required for viability of cells lacking ERMES (Fig. S2 C; compare strains indicated with boxes).

We found that depletion of Mdm12 did not significantly reduce CL synthesis (Fig. 3 C), suggesting ERMES is not required to bring CL precursors to mitochondria. Depletion of Mdm12 in $5 \Delta$ cells did not significantly reduce CL production more than in $5 \Delta$ cells expressing Mdm12, suggesting that the five Vps13-like proteins and ERMES do not play redundant roles in transporting
CL precursors from the ER to mitochondria. Together, these findings suggest that the Vps13-like proteins in yeast do not have an essential role in bulk phospholipid transport to mitochondria.

\section{Csf1 plays a role in PE metabolism}

We wondered whether Csfl, Fmp27, and Hob2 might play a role in PE metabolism since phospholipid transport is necessary for some PE metabolism (Fig. 3 D). PE is produced by two pathways in S. cerevisiae. One makes PE in the ER and requires ethanolamine (Etn; the Kennedy pathway). The second, called the cytidyldiphosphate-diacylglycerol (CDP-DAG) pathway, generates PE primarily outside the ER using enzymes that decarboxylate phosphatidylserine (PS) to form PE (Fig. 3 D). The enzymes are in the mitochondrial inner membrane (Psd1) and endocytic compartments (Psd2; Acoba et al., 2020). It should be noted that 
A

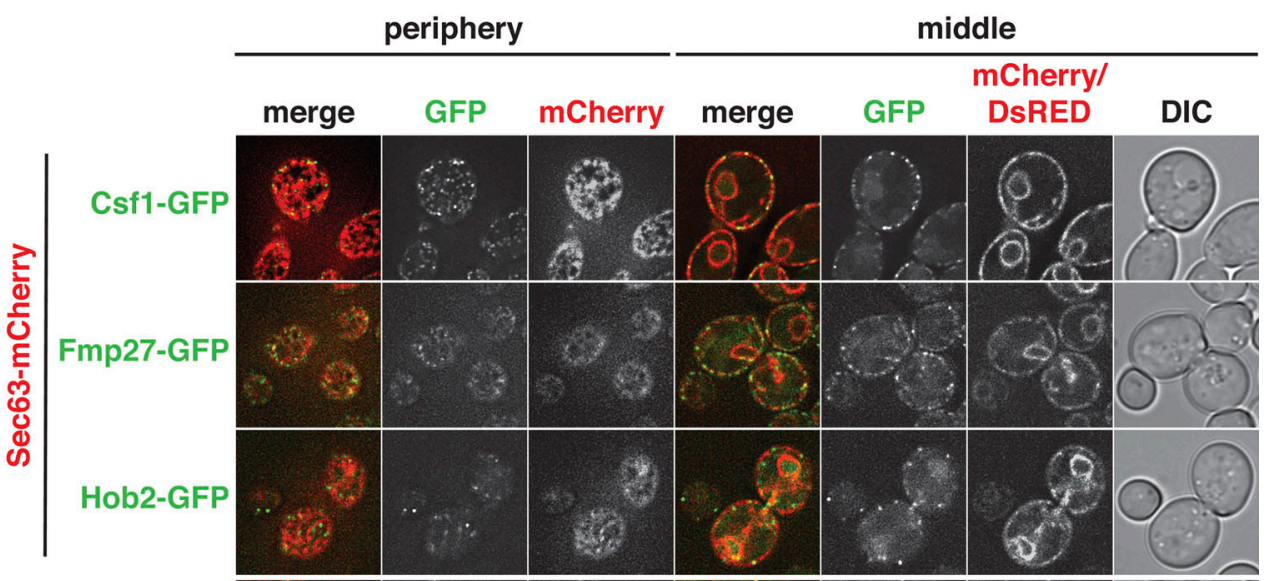

B

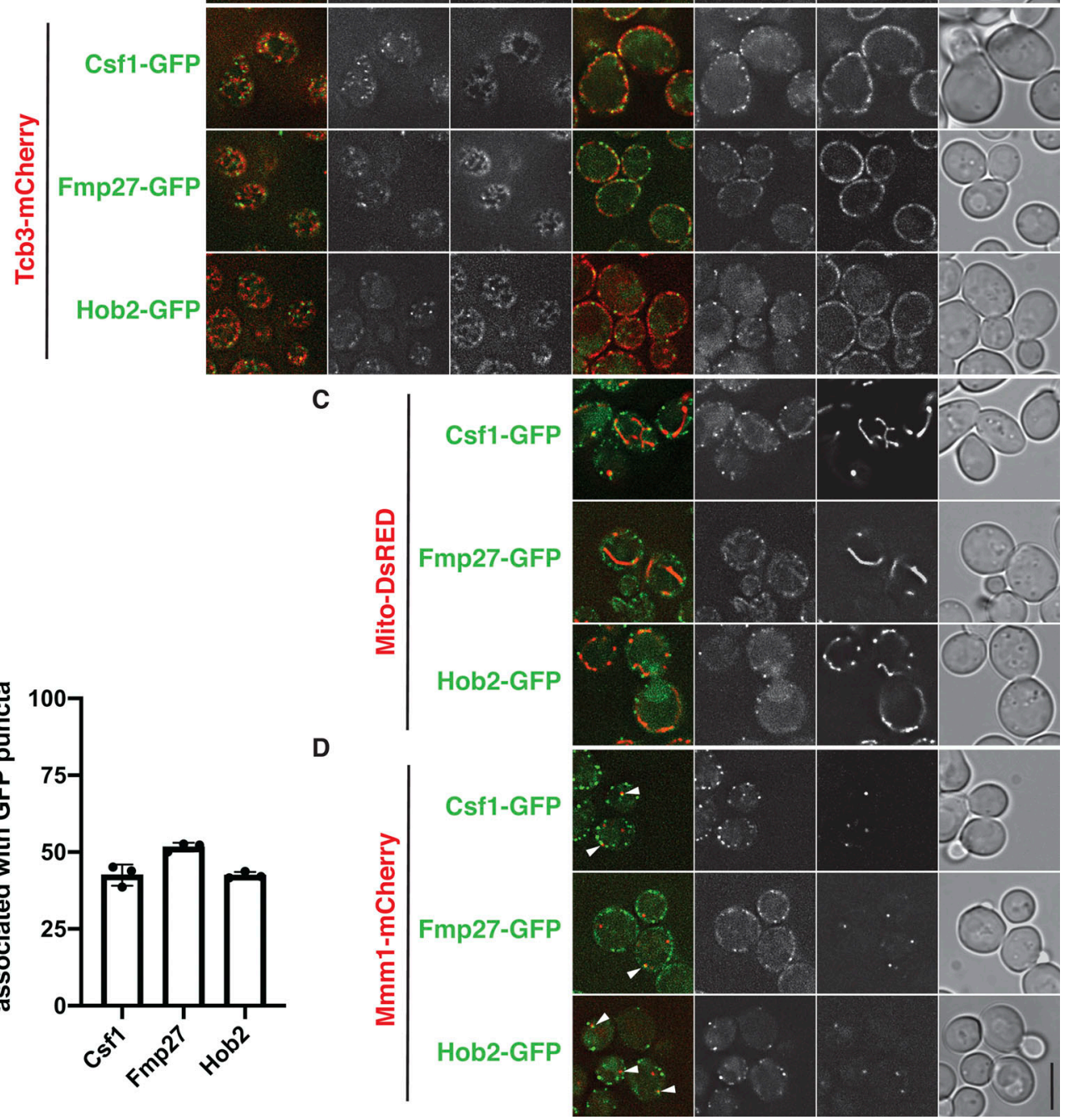

Figure 2. Csf1, Fmp27, and Hob2 localize to ER-PM and ER-mitochondria contact sites. (A-D) Cells were visualized live. Cells expressed GFP fusions to the indicated proteins and the ER marker Sec63-mCherry (A), the ER-PM contact site marker Tcb3-mCherry (B), the mitochondrial marker Mito-DsRed (C), or the ERMES marker Mmm1-mCherry (D). Csf1 and Hob2 are endogenously tagged with seven copies of GFP11, and the strains also express GFP1-10; Fmp27 is endogenously tagged with a single copy of GFP. Cells shown in A and B were visualized by focusing on the middle or periphery of the cells. Bar $=5 \mu \mathrm{m}$. Arrows in D indicate colocalization of ERMES and Csf1, Fmp27, or Hob2. (E) Percentage of Mmm1-mCherry puncta associated with GFP puncta (mean \pm SD of three independent experiments; at least $100 \mathrm{Mmm1-mCherry} \mathrm{puncta} \mathrm{counted} \mathrm{per} \mathrm{experiment).}$ 
A \begin{tabular}{rl|l|l} 
wild-type & \\
$5 \Delta$ & &
\end{tabular}

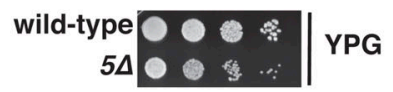
wild-type 000 SC $5 \Delta 00$ " no Etn
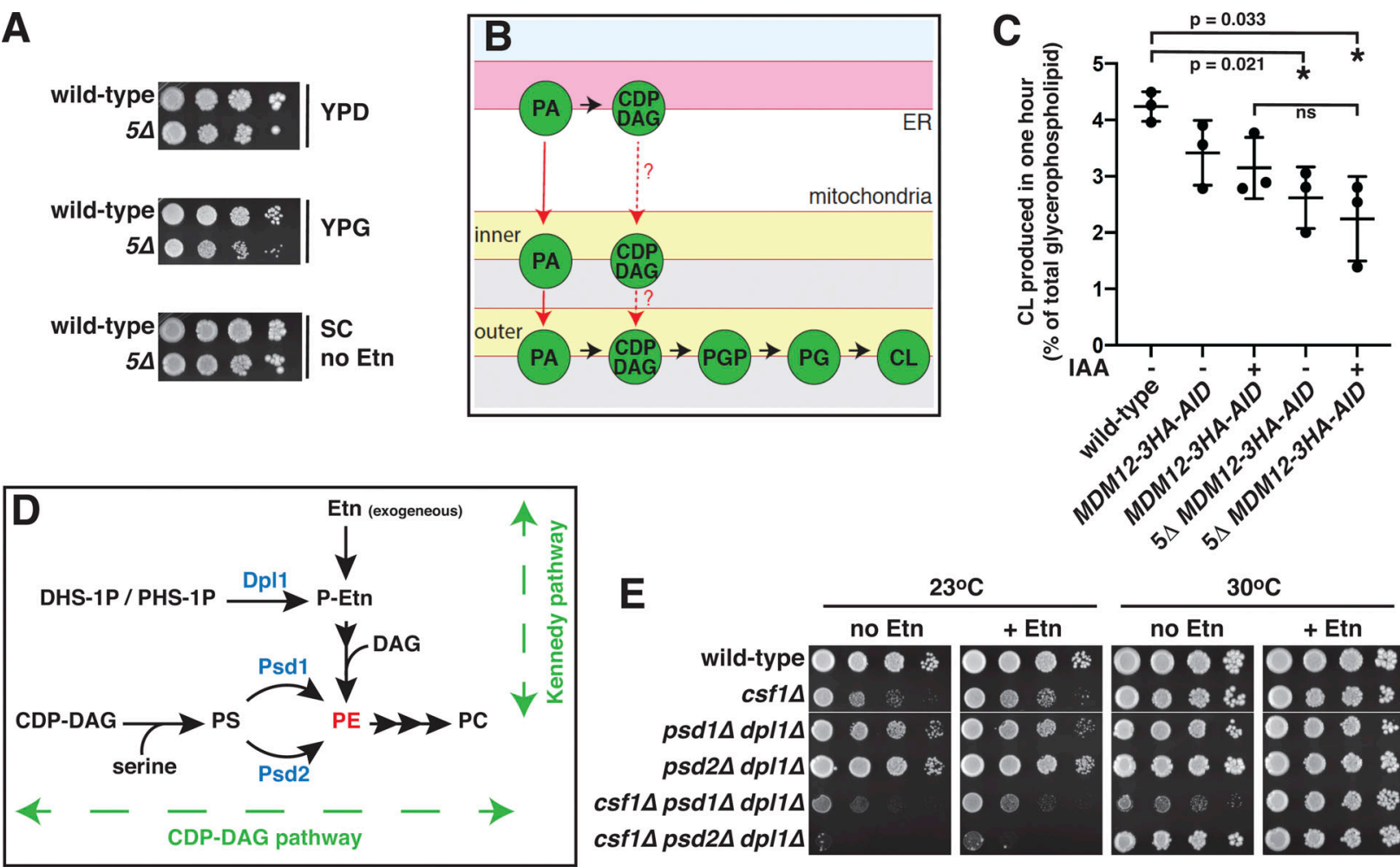

E
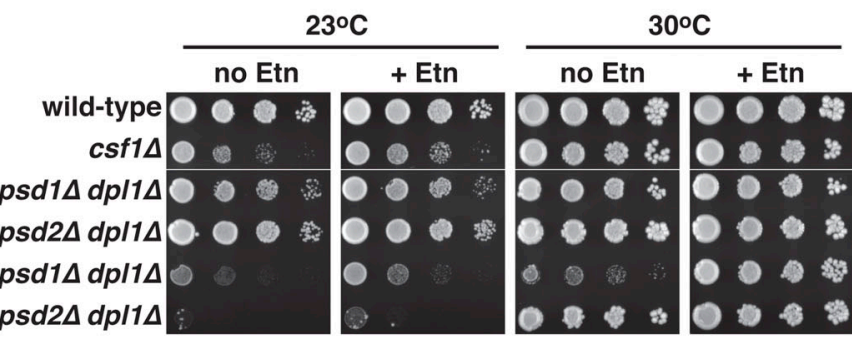

$\mathbf{F}$

O PS

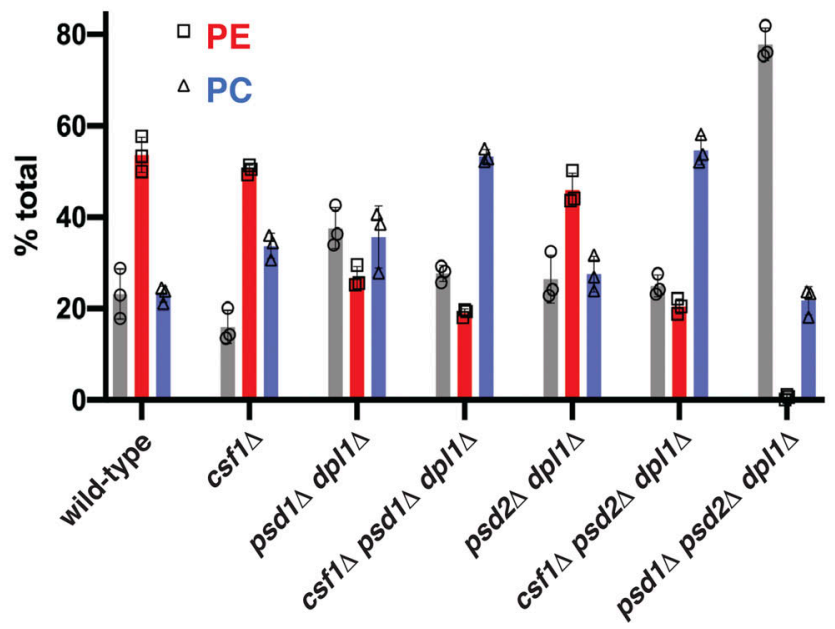

G

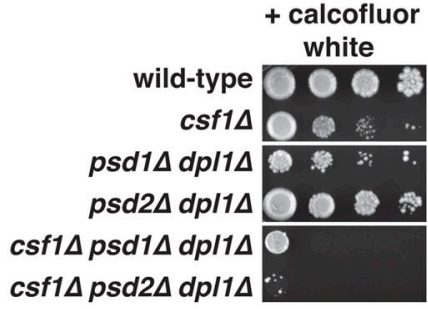

H

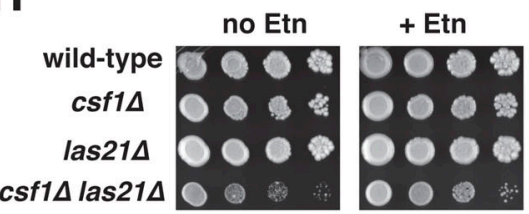

Figure 3. Vps13-like proteins and ERMES are not required to transport CL precursors to mitochondria. (A) 10 -fold serial dilutions of the indicated strains grown on plates with YPD, YPG, or $S C$ without Etn media. The plates were grown at $30^{\circ} \mathrm{C}$ for $3 \mathrm{~d}$. (B) Cartoon showing the biosynthetic pathway of $\mathrm{CL}$ in S. cerevisiae. Phosphatidic acid (PA) and CDP-DAG are produced in the ER membrane (pink). They are transported to the inner membrane of mitochondria (gray), where they are converted to phosphatidylglycerol-phosphate (PGP), phosphatidylglycerol (PG), and CL. (C) The indicated strains were labeled with [ $\left.{ }^{3} \mathrm{H}\right]$ acetate, and the amount of $\mathrm{CL}$ formed in $1 \mathrm{~h}$ was determined. The amount of $\left[{ }^{3} \mathrm{H}\right] \mathrm{CL}$ produced is given as the percentage of the total radiolabeled glycerophospholipids formed. Values from three independent experiments are shown (mean \pm SD). Statistical significance was determined by a Student's $t$ test (twotailed, unpaired); ${ }^{*}, \mathrm{P}<0.05$. Strains with MDM12-3HA-AID express Mdm12 with a C-terminal fusion to 3HA antigen tags and the auxin-inducible degron (AID). Where indicated, the auxin IAA was added to the media 30 min before $\left[{ }^{3} \mathrm{H}\right]$ acetate addition to induce degradation of Mdm12-3HA-AID. (D) Cartoon of the Kennedy and CDP-DAG pathways of PE metabolism. Proteins in blue. Abbreviations not listed in text: DHS-1P, dihydrosphingosine-1 phosphate; PHS-1P, phytosphingosine-1 phosphate. (E) 10-fold serial dilutions of the indicated strains on plates with SC with or without Etn. The plates were grown for $3 \mathrm{~d}$. (F) The indicated strains were labeled with $\left[{ }^{3} \mathrm{H}\right]$ serine for $30 \mathrm{~min}$, and the amount of radiolabeled PS, PE, and PC was determined. The results are the percentage of the three lipids indicated (mean \pm SD from three independent experiments). ( $\mathbf{G}$ and $\mathbf{H}) \mathbf{1 0}$-fold serial dilutions of the indicated strains grown on SC plates containing $10 \mu \mathrm{g} / \mathrm{ml}$ calcofluor white $(\mathrm{G})$ or SC with or without Etn $(\mathrm{H})$. The plates were grown in $30^{\circ} \mathrm{C}$ for $3 \mathrm{~d}$. 
a fraction of Psdl is in the ER; therefore, the CDP-DAG pathway may produce some PE in the ER (Friedman et al., 2018). PE produced by the Kennedy pathway requires exogenous Etn or P-Etn produced by dihydrosphingosine phosphate lyase (Dpll; Fig. $3 \mathrm{D}$ ). Cells lacking Dpll and grown in media that do not contain Etn can only make PE using the CDP-DAG pathway.

We found Csfl plays a role in the CDP-DAG pathway of PE metabolism. Cells lacking Csfl, Dpl1, and either Psd1 or Psd2 grow poorly in a medium without Etn (Fig. 4 E). The strains grew normally when Etn was added to the medium, suggesting Csfl is particularly important for the CDP-DAG pathway of PE metabolism. A role for Csfl in PE metabolism has also been suggested by a recent preprint from the Kornmann group (John Peter et al., 2021 Preprint). The growth of cells lacking Fmp27 or Hob2 was not reduced in a medium lacking Etn (not shown); therefore, we focused on Csfl.

To determine whether PE metabolism by the CDP-DAG pathway is impaired in cells lacking Csfl, we labeled cells with $\left[{ }^{3} \mathrm{H}\right]$ serine for $1 \mathrm{~h}$. Cells use $\left[{ }^{3} \mathrm{H}\right]$ serine to produce $\left[{ }^{3} \mathrm{H}\right] \mathrm{PS}$, which can be converted into PE and, in turn, into phosphatidylcholine (PC) by the CDP-DAG pathway (Fig. 3 D). We previously showed that following $\left[{ }^{3} \mathrm{H}\right]$ serine addition to the medium of growing cells, radiolabeled PS, PE, and PC are produced at linear rates (Raychaudhuri and Prinz, 2008). The labelings were done in cells lacking Dpll since this prevents the production of radiolabeled P-Etn that could be used to make PE. Surprisingly, we found cells lacking Csfl have no defect in producing PS (Fig. S2 E) or converting it into PE and PC (Fig. 3 F). This suggests they do not have significant defects in transporting PS to Psdl and Psd2 or returning the PE produced by these enzymes back to the ER, where it is converted to PC. Together, these findings indicate Csfl has a role in PE metabolism but does not affect the production of PS, PE, or PC.

\section{Cells lacking Csf1 do not have defects in autophagy}

Since Csfl plays a role in PE metabolism but not in the production of $\mathrm{PE}$ or the conversion of $\mathrm{PE}$ to $\mathrm{PC}$, we wondered whether Csfl is necessary for a PE-dependent process. One is autophagy, which requires the conjugation of $\mathrm{PE}$ to the protein Atg8, a protein that plays a role in autophagosome formation (Ichimura et al., 2000). During autophagy, Atg8 is conjugated to $\mathrm{PE}$ (forming Atg8-PE) and delivered to the vacuole, where it is degraded. When GFP is fused to the N-terminus of Atg8, the Atg8 portion of the fusion is degraded in the vacuole during autophagy while the GFP portion remains relatively stable; thus, the presence of free GFP in cells expressing GFP-Atg8 indicates it was trafficked to the vacuole during autophagy (Shintani and Klionsky, 2004). If Csfl provides the PE necessary for GFP-Atg8PE production, cells lacking Csfl would have reduced levels of free GFP following autophagy induction.

We induced autophagy in cells expressing GFP-Atg8 by shifting them to nitrogen starvation medium for $3 \mathrm{~h}$. This caused the appearance of free GFP in WT cells but not in cells lacking Atg2 or Atg1, a protein kinase required for autophagy (Fig. S2 F). The production of free GFP was not affected in cells lacking Csfl (Fig. S2 F), indicating Csfl probably does not play a role in providing PE to produce Atg8-PE.

\section{GPI anchor precursors lacking P-Etn accumulate in cells lacking Csf1}

We wondered whether the role of Csfl in PE metabolism could be to support GPI anchor biosynthesis, since the biosynthesis of each anchor requires three PE molecules (Fig. 4 A). Consistent with this possibility, cells lacking Csfl have a defect in trafficking a GPI-anchored protein (Copic et al., 2009), and the genetic profile of CSF1 is like that of many genes required for GPI anchor synthesis (https://thecellmap.org/). Cells with defects in GPI anchor synthesis are hypersensitive to the antifungal calcofluor white (Benghezal et al., 1995), and we found cells lacking Csfl are also hypersensitive (Fig. $3 \mathrm{G}$ ). In addition, there is a negative genetic interaction between CSF1 and LAS21 (Fig. $3 \mathrm{H}$ ), which encodes one of the three Etn phosphotransferases involved in GPI anchor biosynthesis (Fig. 4 A). Together, these findings implicate a potential function of Csfl in GPI anchor production.

We speculated that if Csfl plays a role in providing PE for GPI anchor biogenesis, nascent GPI anchors lacking P-Etn would accumulate in cells lacking Csfl. To test this, we labeled various strains with $\left[{ }^{3} \mathrm{H}\right]$ inositol, extracted phospholipids, and separated them by TLC as previously described (Flury et al., 2000). This study showed that GPI anchor intermediates lacking P-Etn accumulate in cells with mutations in any of the three genes encoding the Etn phosphotransferases were required to produce GPI anchors: MCD4, GPI13, and LAS21 (Fig. 4 A). Cells with a conditional gpil3-5 allele accumulate the lipid 031a, a GPI anchor precursor that lacks P-Etn (Fig. 4 B; Flury et al., 2000). We found lipid 031a also accumulates in cells lacking Csfl and proteins in the CDP-DAG pathway of PE biosynthesis, Psdl or Psd2 (Fig. 3 D; and Fig. 4, C and D). We noted, however, that cells lacking only Csfl do not accumulate lipid 031a. Since the labeling experiments were done at $30^{\circ} \mathrm{C}$, we wondered whether cells lacking Csfl would accumulate lipid 031a when grown at $23^{\circ} \mathrm{C}$, which is known to reduce growth of these cells (Tokai et al., 2000), a finding we confirmed (Fig. $3 \mathrm{E}$ ). Indeed, at $23^{\circ} \mathrm{C}$, the cells lacking Csfl accumulated significantly more lipid 031a than WT cells (Fig. 4 E), suggesting Csfl supports GPI anchor biosynthesis.

To further investigate which are the Csfl-dependent steps in GPI anchor biosynthesis, we characterized strains lacking Las21, which accumulate the GPI anchor precursor M4 that has two P-Etns of the three P-Etns normally added to GPI anchors (Fig. 4 B; Flury et al., 2000). We confirmed this finding and discovered that cells lacking Csfl also accumulate lipid M4, though not as much as cells lacking Las21 (Fig. 4, F and G). Interestingly, cells lacking both Las 21 and Csfl have reduced amounts of lipid M4 but more lipid 031a (Fig. $4 \mathrm{G}$ ). This finding suggests Csfl is necessary for a step in GPI synthesis before the one catalyzed by Las21, probably the addition of one or both of the first two P-Etns added to GPI anchors. Together, these findings suggest Csfl is necessary to support the addition of P-Etn to GPI anchor precursors.

\section{Csf1 may channel PE to the Etn phosphotransferase Mcd4}

Since Csfl is likely to be a tube-forming LTP and localizes to MCSs between the ER and other organelles, we hypothesized that it transports PE to the ER to support GPI anchor biogenesis 


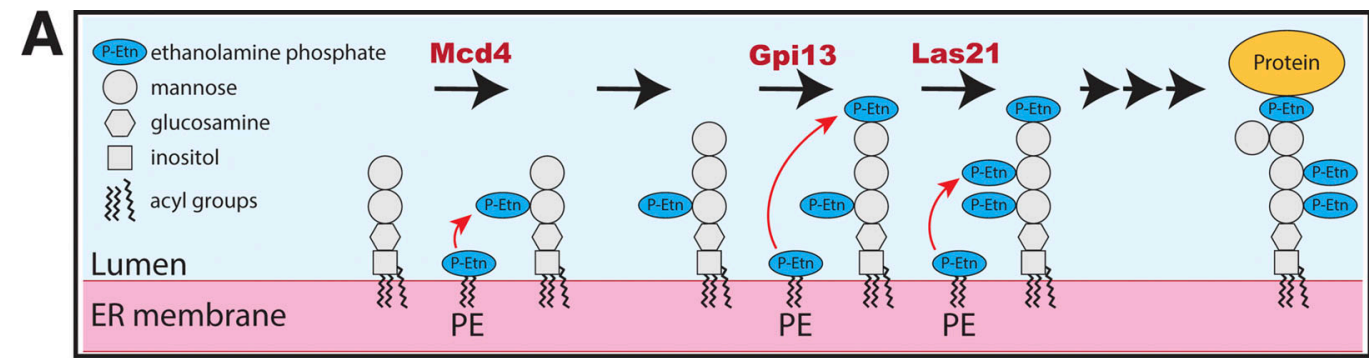

B
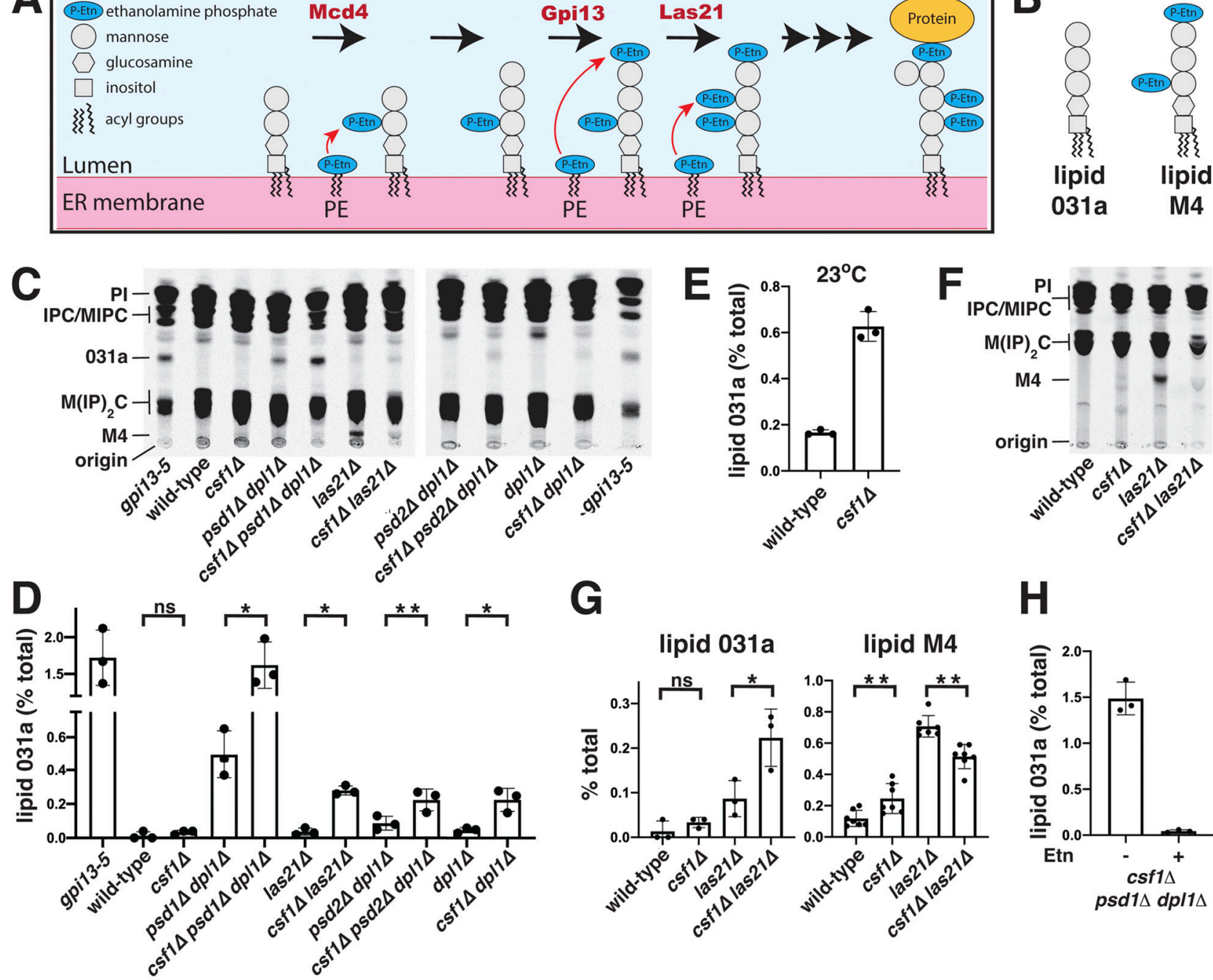

G

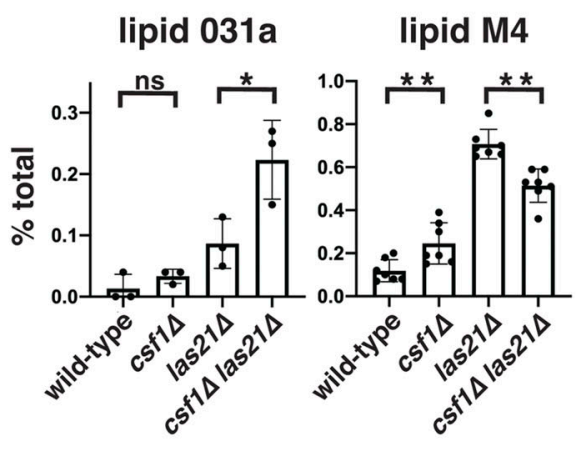

H
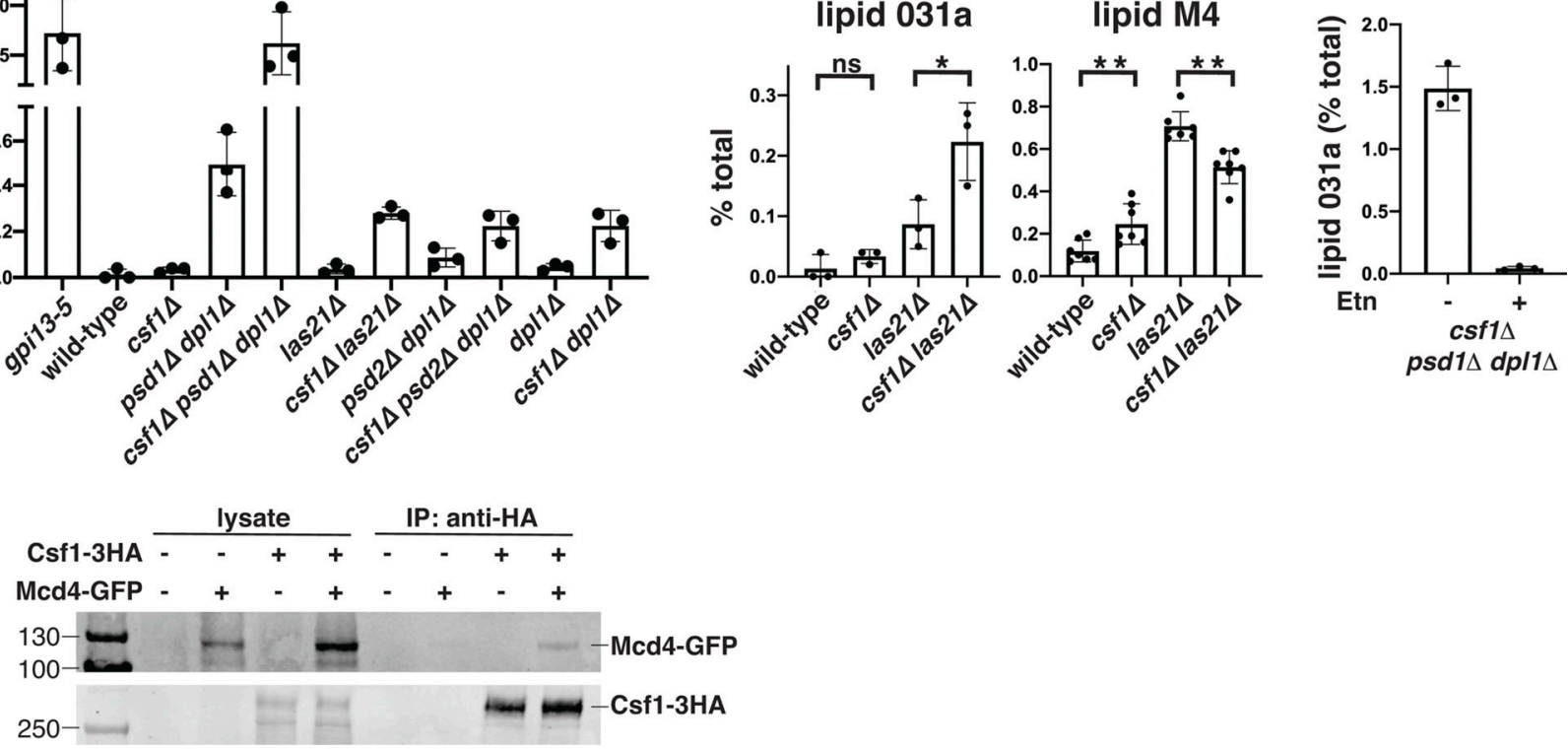

Figure 4. Cells lacking Csf1 accumulate nascent GPI anchors lacking P-Etn. (A) Cartoon of the three steps in GPI anchor biogenesis where P-Etn is added to nascent GPI anchors. The three Etn phosphotransferases are in red. (B) GPI anchor intermediates (symbols as in A). (C) The indicated strains were labeled with $\left[{ }^{3} \mathrm{H}\right]$ inositol for $2 \mathrm{~h}$, and lipids were extracted and separated by TLC. All strains were grown in $\mathrm{SC}$ at $30^{\circ} \mathrm{C}$ except gpi13-5 cells, which were grown at $23^{\circ} \mathrm{C}$ and shifted to $37^{\circ} \mathrm{C}$ for 30 min before labeling. Abbreviations not listed in text: IPC, inositol-phosphoryl-ceramide; MIPC, mannosyl-inositol-phosphoryl-ceramide; $\mathrm{M}(\mathrm{IP})_{2} \mathrm{C}$, mannosyl diinositol-phosphoryl-ceramide; PI, phosphatidylinositol. (D) Quantification of the amount of lipid 031a formed in the experiments shown in C. Mean \pm SD of three independent experiments. Statistical significance determined by Student's $t$ test (two-tailed, paired). ${ }^{*}, P<0.05 ;{ }^{* *}, P<0.005$. (E) Quantification of the amount of lipid 031a formed in the indicated strains as described in C and D, except the strains were grown at $23^{\circ} \mathrm{C}$ and labeled for $24 \mathrm{~h}($ mean $\pm \mathrm{SD})$. (F) The indicated strains were labeled with $\left[{ }^{3} \mathrm{H}\right]$ inositol as described in $\mathrm{C}$, except that lipids were separated by TLC using a solvent system that resolves lipid M4. (G) Quantification of the amount of lipid M4 formed as in the experiments shown in $F$ is shown in the right panel. The left panel shows the same data given in D. Mean \pm SD of three independent experiments. Statistical significance determined with a Student's $t$ test (two-tailed, paired). ${ }^{*}, P<$ $0.05{ }^{* *}, \mathrm{P}<0.005$. (H) Quantification of the amount of lipid 031a formed in the indicated strains as described in C and D, except grown in SC media either with or without Etn (mean \pm SD). (I) Lysates from cell expressing Csf1-3HA and Mcd4-GFP as indicated were incubated with magnetic beads conjugated to an antiHA antibody. Whole-cell lysates and immunoprecipitated (IP) proteins were separated by SDS-PAGE and immunoblotted with anti-HA and anti-GFP antibodies. Numbers are size of molecular weight markers $(\mathrm{kDa})$. Representative blots from three independent experiments are shown. Source data are available for this figure: SourceData F4. 
and that this is particularly important in cells where most $\mathrm{PE}$ is made outside the ER. If this is correct, production of PE in the ER by the Kennedy pathway might bypass the role of Csfl in GPI anchor biosynthesis. We found that addition of Etn to cells lacking Csfl, Psdl, and Dpll eliminated accumulation of lipid 031a found in this strain when it is grown without exogenous Etn (Fig. $4 \mathrm{H}$ ). Thus, PE production in the ER supports efficient GPI anchor synthesis in cells lacking Csfl.

These findings suggest that in growth conditions where most $P E$ is synthesized outside the ER, there is insufficient PE in the ER to support robust GPI anchor production, perhaps because most PE in the ER is converted to PC. If this is correct, Csfl may prevent this problem by channeling PE outside the ER to the GPI synthesis machinery. Consistent with this, we used coimmunoprecipitation to show that Csfl interacts with Mcd4, the Etn phosphotransferase that adds the first P-Etn to nascent GPI anchors (Fig. 4 A). Endogenous Csfl was tagged with three copies of the HA epitope (Csfl-3HA), and we confirmed Csfl-3HA is functional (Fig. S2 B). We immunoprecipitated Csfl-3HA and found it coimmunoprecipitates with Mcd4 fused to GFP (Mcd4GFP) expressed from a plasmid under the native MCD4 promoter (Fig. 4 I). Together, these findings suggest Csfl channels PE to Mcd4 to support GPI anchor synthesis. This channeling may not be necessary when significant amounts of PE are produced in the ER, which probably increases the amount of PE in the ER available for GPI anchor production.

\section{Csf1 homologues in higher eukaryotes participate in GPI- anchored protein biogenesis}

Csfl has homologues in higher eukaryotes. We wondered whether they are necessary for efficient GPI anchor biogenesis. To test this, we determined whether knocking down or knocking out the homologues in human cells and Caenorhabditis elegans decreases the amount of GPI-anchored proteins on the surface of cells.

The human homologue of Csfl is KIAA1109, or TWEEK. Mutations in TWEEK were recently shown to cause a disorder of brain development and arthrogryposis named AlkurayaKučinskas syndrome (Gueneau et al., 2018). We used shRNA to knock down expression of TWEEK and the GPI biogenesis gene GPI Anchor Attachment 1 (GPAA1) in human embryonic kidney 293 (HEK-293) cells. GPAA1 is required to add proteins to GPI anchors (Hiroi et al., 1998). We used FACS to show that knockdown of GPAAl reduces the amount of the GPI-anchored protein CD55 on the surface of cells (Fig. 5 A). Knockdown of TWEEK with either of two shRNAs that target different sequences similarly reduced the amount of CD55 on the surface of the HEK-293 cells (Fig. 5, B and C). We used quantitative real-time RT-PCR (qRT-PCR) to confirm the knockdown of TWEEK and GPAAl (Fig. S3 A).

The $C$. elegans genome encodes a single KIAA1109/TWEEK orthologue, lpd-3. Four lpd-3 transcripts have been identified that vary by only a few amino acids. We generated a null allele of lpd-3 that lacks exons 1-6 (and intervening introns) and introduces three tandem stop codons in each reading frame (Fig. S3, B-D). Homozygous lpd-3 N- $\Delta$ animals were validated by PCR and Sanger sequencing (Fig. S3, C and D). The lpd-3 N-A animals had shortened gonads and a deformed germline (Fig. S3 E). Similar defects are found in GPI anchor-synthesis mutants (Murata et al., 2012). We used fluorescein-labeled proaerolysin (FLAER), which binds GPI anchors, to detect GPI-anchored proteins in the PM of WT and lpd-3 N- $\Delta$ embryos. Before the first mitotic cell division, embryos have little FLAER signal at the PM (not shown). At the two-cell stage, WT embryos had significant FLAER signal in the PM at the cleavage furrow, while lpd-3 N- $\Delta$ had significantly less (Fig. 5, D and E). This was also true of four-cell-stage embryos (Fig. S3 F). Taken together, these findings indicate that KIAA1109/TWEEK and LPD-3 play roles in the biogenesis of GPI-anchored proteins and suggest they facilitate GPI anchor biogenesis like Csfl.

\section{Conclusions}

We propose that Csfl channels PE to Mcd4 in the ER to support GPI anchor production (Fig. $5 \mathrm{G}$ ). This is consistent with work from the Menon group that suggested PE used for GPI synthesis is preferentially derived from PE produced outside the ER in mitochondria (Vidugiriene et al., 1999). Our results suggest the Csfl homologues KIAA1109/TWEEK and Lpd-3 support GPI anchor production and probably have the same function as Csfl. Since humans (and other mammals) cannot synthesize Etn and derive it mostly from dietary lipids (Calzada et al., 2016; Patel and Witt, 2017), KIAA1109/TWEEK may be particularly important for GPI biosynthesis when dietary sources of Etn are low. The role of KIAA1109/TWEEK in GPI anchor biogenesis in Alkuraya-Kučinskas syndrome should be investigated.

Our findings strongly suggest that Csfl transports PE to the ER. However, we cannot rule out that Csfl indirectly affects GPI anchor synthesis by transporting another lipid or regulating GPI anchor synthesis proteins such as Mcd4. Assuming Csfl does transport PE to the ER, it surprisingly is not necessary to bring PE to the ER to support PC synthesis but instead seems to direct PE to GPI anchor biosynthesis. It is likely that most PE in the ER is used to produce $\mathrm{PC}$, and Csfl may ensure there is sufficient $\mathrm{PE}$ to support GPI anchor production when PE levels are low in the ER (Fig. $5 \mathrm{G}$ ). Understanding the mechanism and regulation of lipid transport by tube-forming LTPs such as Csfl is an important challenge for the future.

\section{Materials and methods}

\section{Strains and cell line}

The yeast strains used in this study are listed in Table S1. Strains were grown in SC media containing $2 \%$ glucose (Sigma-Aldrich), $0.67 \%$ nitrogen base (BD), and an appropriate amino acid dropout mix (US Biological); or YPD media containing $1 \%$ yeast extract $(\mathrm{BD}), 2 \%$ peptone (BD), and $2 \%$ glucose; or YPG media containing $1 \%$ yeast extract $(\mathrm{BD}), 2 \%$ peptone $(\mathrm{BD})$, and $3 \%$ glycerol; or nitrogen starvation medium (SD-N) media containing $2 \%$ glucose (Sigma-Aldrich) and $0.67 \%$ nitrogen base (BD). Where indicated, Etn (Sigma-Aldrich) was added to media to a final concentration of $1 \mathrm{mM}$ from a $1 \mathrm{M}$ stock.

The C. elegans strains used in this study were N2 (Bristol, WT) and AG645 (lpd-3[av254]V. CRISPR/Cas9 Edit, deletion of exons 1-6 and intervening introns). 

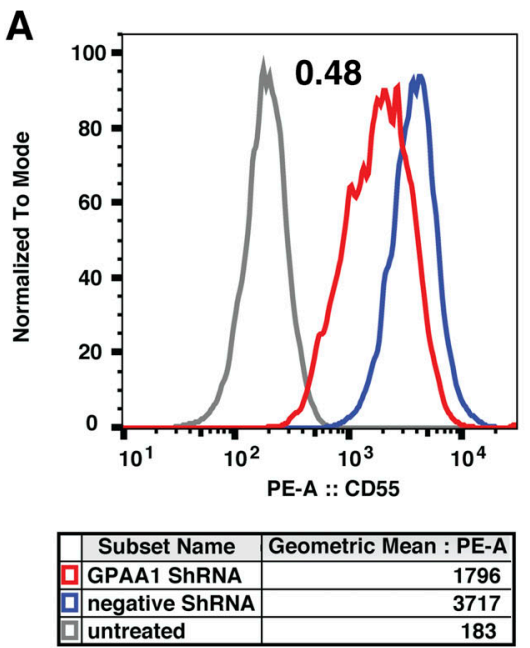

D
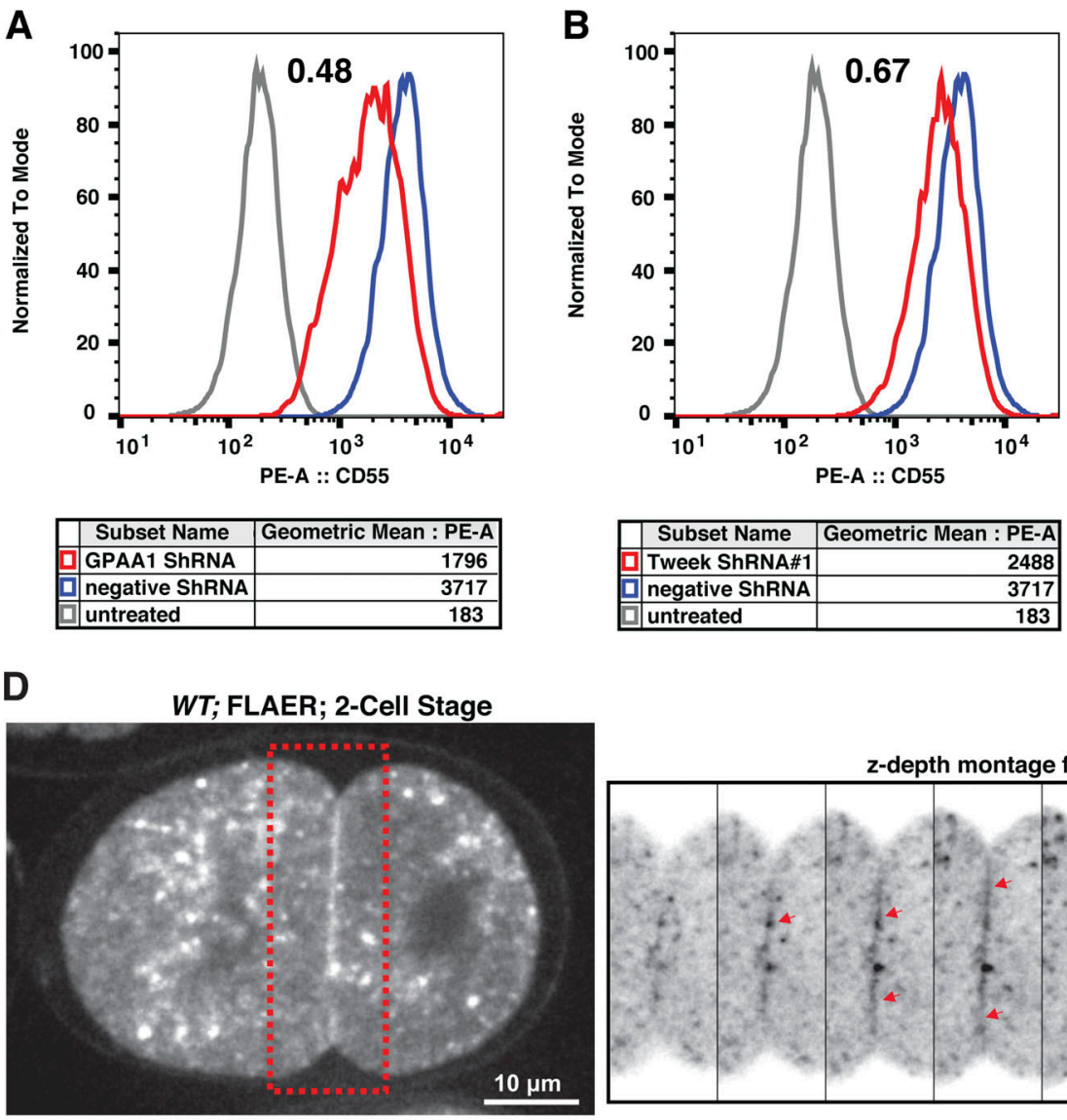

C

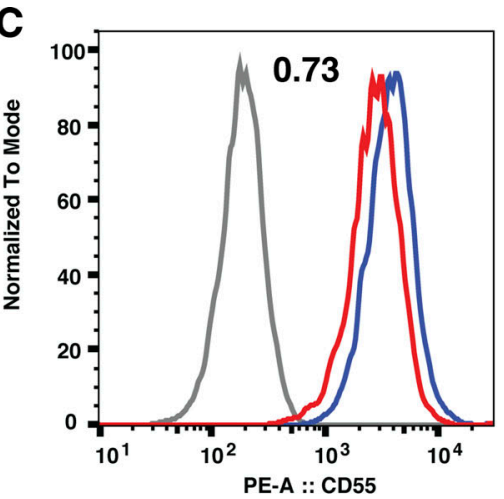

\begin{tabular}{|l|l|r|}
\hline & Subset Name & Geometric Mean : PE-A \\
\hline 0 & Tweek ShRNA\#2 & $\mathbf{2 7 1 0}$ \\
\hline \hline & negative ShRNA & 3717 \\
\hline 0 & untreated & 183 \\
\hline
\end{tabular}

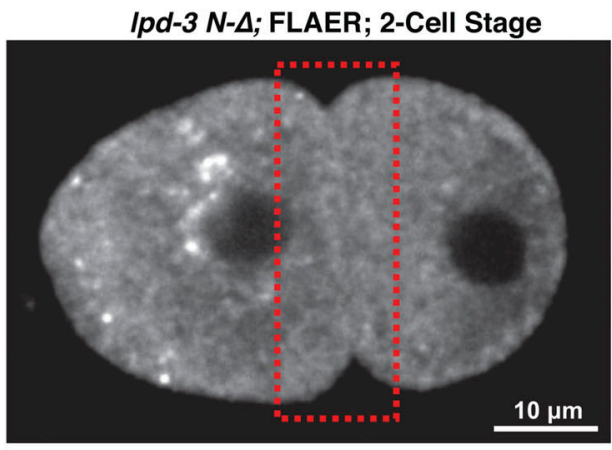

E

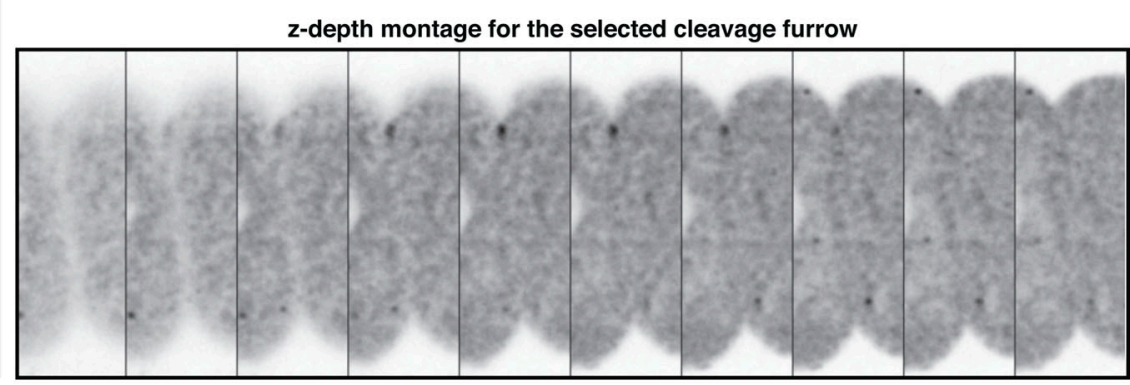

$\mathbf{F}$
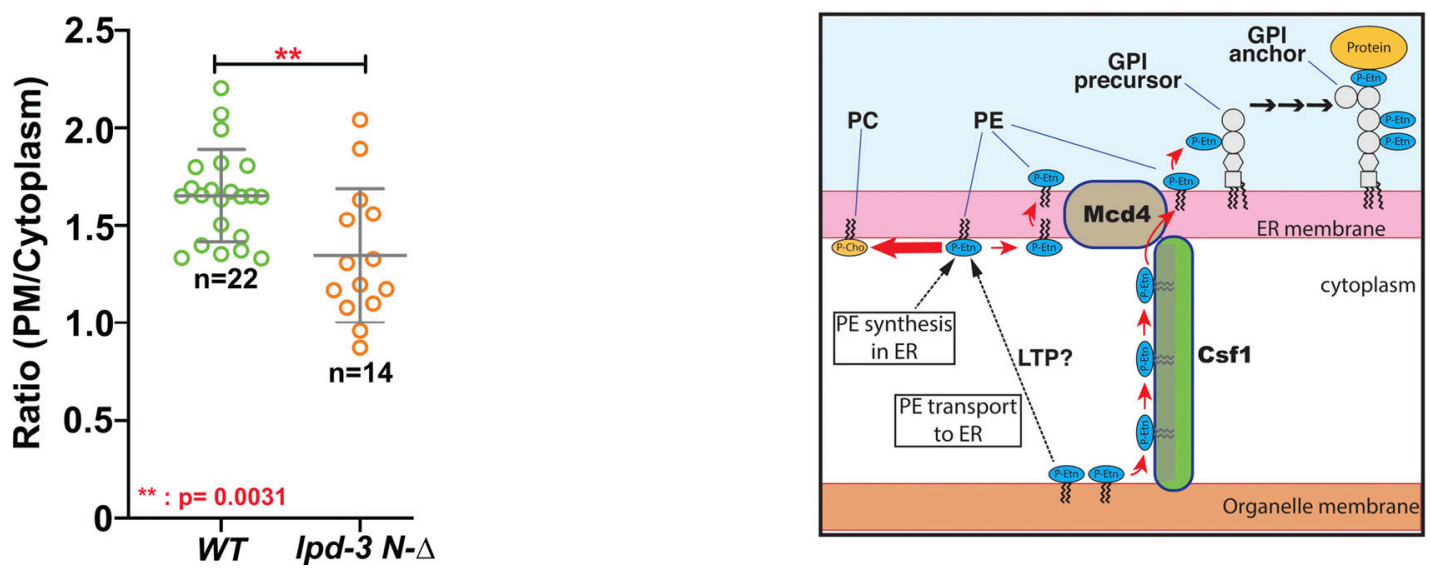

Figure 5. Csf1 homologues play a role in biogenesis of GPI-anchored proteins. (A-C) Flow-cytometry analysis of HEK-293 cells expressing shRNA targeting GPAA1 (A) or two different shRNAs targeting KIAA1109/TWEEK (B and C) using anti-CD55 antibody conjugated to R-phycoerythrin (PE-A). 10,000 cells 
were counted, and the geometric mean for negative shRNA (blue), gene targeted by shRNA (red), and untreated (no antibody, gray) shown. Ratio of the geometric mean of fluorescence intensity of the cells treated with shRNA to the targeted genes to negative control are shown in each panel. (D) FLAER staining of two-cell-stage $C$. elegans embryos from WT and lpd-3 N- $\Delta$ animals. Right panels show z-depth montages of the cleave furrows indicated by the red boxes. Bars $=10 \mu \mathrm{m}$. (E) Quantification of FLAER signals in the cleave furrow of two-cell embryos as shown in D ( $n=$ number of tested embryos). P value determined by Student's $t$ test (two-tailed, unpaired). (F) Model of PE channeling to Mcd4 at ER contact sites. Red arrows indicate movement or metabolism of PE.

HEK-293 cells were cultured with DMEM (4.5 g/liter D-Glucose + L Glutamine; Gibco), 10\% FBS heat-inactivated (Quality Biological), and penicillin and streptomycin (Gibco) in incubator at $37^{\circ} \mathrm{C} 5 \% \mathrm{CO}_{2}$.

\section{Serial dilutions to assess growth}

Cells were grown in the indicated medium to logarithmic growth phase, washed twice with medium, and adjusted to an $\mathrm{OD}_{600}$ of 0.5-1.0. 10-fold serial dilutions were prepared in the medium and spotted onto plates. The plates were incubated for $3 \mathrm{~d}$ at the indicated temperature.

\section{Microscopy of yeast}

Cells were imaged live at $30^{\circ} \mathrm{C}$ using DeltaVision Spectris system (Applied Precision), with a wide-field inverted epifluorescence microscope (IX70; Olympus), 100× objective, and a chargecoupled device CoolSNAP HQ camera (Photometrics). Images were deconvolved using the conserved ratio method, 10 cycles, with softWoRx (Applied Precision).

\section{Labeling cells with $\left[{ }^{3} \mathrm{H}\right]$ serine and analysis of glycerophospholipids}

Cells were grown to saturation at $30^{\circ} \mathrm{C}$ in SC lacking serine (Complete Supplementation Mix; Sunrise Science Products) with $1 \mathrm{mM}$ Etn. $2 \mathrm{ml}$ of a saturated culture was added to $50 \mathrm{ml}$ of fresh medium. The cells were grown for $4.5 \mathrm{~h}$, washed twice with water, and resuspended in $50 \mathrm{ml}$ of fresh medium lacking Etn at an $\mathrm{OD}_{600}$ of $0.2-0.4$. After $3 \mathrm{~h}$ of growth, $0.1 \mathrm{mCi}$ of $\left[3-{ }^{3} \mathrm{H}\right]$ serine (American Radiolabeled Chemicals) was added, and the cells were grown for $1 \mathrm{~h}$. After labeling, the cells were cooled to $4^{\circ} \mathrm{C}$, washed once with water, and lysed in a Precellys 24 homogenizer. Lipids were extracted and separated by TLC as described (Kannan et al., 2017). TLC plates were scanned on a RITA Star thin-layer analyzer (Raytest) to the relative radioactive signal from each phospholipid class.

\section{Labeling cells with $\left[{ }^{3} \mathrm{H}\right]$ acetate and analysis of glycerophospholipids}

Cells were grown to saturation at $30^{\circ} \mathrm{C}$ in YPD. $250-400 \mu \mathrm{l}$ of the saturated cultures was added to $10 \mathrm{ml}$ of fresh YPD. The cultures were grown at $30^{\circ} \mathrm{C}$ for $4 \mathrm{~h} ; 5 \mu \mathrm{l}$ of indole-3-acetic acid (IAA; SigmaAldrich; stock = $1 \mathrm{M}$ in ethanol) or ethanol was added; after $30 \mathrm{~min}$, $50 \mu \mathrm{Ci}\left[{ }^{3} \mathrm{H}\right]$ acetate (American Radiolabeled Chemicals) was added; and the cultures were grown for $1 \mathrm{~h}$. The cells were added to ice-cold water and washed once with water, and the lipids were extracted and analyzed as described in the previous section.

\section{Measurement of autophagy induction}

Cells were grown to mid-logarithmic phase in SC at $30^{\circ} \mathrm{C}$, washed three times with SD-N medium, and incubated for $3 \mathrm{~h}$ in
$\mathrm{SD}-\mathrm{N}$ at $30^{\circ} \mathrm{C}$. At the indicated times, $3 \mathrm{OD}_{600}$ units of cells were withdrawn. The cells were pelleted, resuspended in $50 \mu \mathrm{l}$ of $1.85 \mathrm{M} \mathrm{NaOH}$, incubated on ice for $10 \mathrm{~min}$, mixed with $125 \mu \mathrm{l}$ of $20 \%$ trichloroacetic acid, and incubated on ice for $30 \mathrm{~min}$. The precipitated proteins were separated by SDS-PAGE and immunoblotted with anti-GFP antibodies (Roche).

\section{Measurement of GPI anchor intermediates}

GPI anchor intermediates were measured as previously described (Flury et al., 2000; Reggiori et al., 1997). Briefly, 2.5 $\mathrm{OD}_{600}$ units of logarithmic growth phase cells were suspended in $250 \mu \mathrm{l} \mathrm{SC}$ containing $25 \mu \mathrm{Ci}$ myo- $\left[1,2-{ }^{3} \mathrm{H}(\mathrm{N})\right]$ inositol (American Radiolabeled Chemicals). The cells were incubated for $40 \mathrm{~min}$ at $30^{\circ} \mathrm{C}, 750 \mu \mathrm{l} \mathrm{SC}$ was added, and the cells were incubated for $1 \mathrm{~h}$ and $20 \mathrm{~min}$. The cells were pelleted and frozen at $-80^{\circ} \mathrm{C}$. The gpil3-5 cells were grown at $23^{\circ} \mathrm{C}$ to logarithmic growth phase in SC, shifted to $37^{\circ} \mathrm{C}$ for $30 \mathrm{~min}$, and then labeled as described except at $37^{\circ} \mathrm{C}$. Cells labeled at $23^{\circ} \mathrm{C}$ were incubated with $50 \mu \mathrm{Ci}$ myo- $\left[1,2-{ }^{3} \mathrm{H}(\mathrm{N})\right]$ inositol for $24 \mathrm{~h}$.

The labeled cells were thawed and resuspended in $30 \mu \mathrm{l}$ icecold breaking buffer (60 mM NaPi, $\mathrm{pH} 8,44 \mathrm{mM} \mathrm{NaCl}$, and $55 \mathrm{mM}$ glucose). $300 \mu \mathrm{l}$ glass beads (425-600 mm; Sigma-Aldrich) and $300 \mu \mathrm{CHCl}_{3}$ :methanol $(\mathrm{MeOH}): \mathrm{H}_{2} \mathrm{O}$ (10:10:3) were added, and the cells were lysed in a Precellys 24 homogenizer at $4^{\circ} \mathrm{C}(3 \times 6,000 \mathrm{rpm}$ for $30 \mathrm{~s}) .900 \mu \mathrm{l} \mathrm{CHCl} / \mathrm{MeOH} / \mathrm{H}_{2} \mathrm{O}(10: 10: 3)$ was added, and the samples were vortexed and centrifuged for $5 \mathrm{~min}$ at $15,000 \times \mathrm{g}$ at room temperature. The supernatants were dried in a SpeedVac. The samples were resuspended in $100 \mu \mathrm{l}$ of water-saturated butanol and desalted by adding $50 \mu \mathrm{l}$ of water, vortexed, and centrifuged for $3 \mathrm{~min}$ at $15,000 \times \mathrm{g}$ at room temperature. The upper phase was removed and saved. The bottom phase was reextracted with $100 \mu \mathrm{l}$ of water-saturated butanol, and the pooled upper phases were dried under $\mathrm{N}_{2}$.

The samples were resuspended with $40 \mu \mathrm{C} \mathrm{CHCl} 3 / \mathrm{MeOH} / \mathrm{H}_{2} \mathrm{O}$ (10:10:3) and spotted onto Silica gel 60 TLC plates (Merck). The plates were developed for $1 \mathrm{~h} 20 \mathrm{~min}$ in either $\mathrm{CHCl}_{3}: \mathrm{MeOH}$ : $0.25 \% \mathrm{KCl}(55: 50: 10)$ to quantify lipid 031a or $\mathrm{CHCl}_{3}: \mathrm{MeOH}: \mathrm{H}_{2} \mathrm{O}$ (10:10:3) to quantify the GPI precursor lipid M4. The TLC plates were scanned on a RITA Star thin-layer analyzer (Raytest) to quantify radioactivity or visualized with a Typhoon FLA 9500 laser scanner (GE Healthcare).

\section{Coimmunoprecipitation}

Cells were grown to logarithmic growth phase, and $150 \mathrm{OD}_{600}$ units of cells were washed once with ice-cold water, pelleted, and resuspended in $600 \mu \mathrm{l}$ of lysis buffer (50 mM Tris, $\mathrm{pH} 7.4$, $150 \mathrm{mM} \mathrm{NaCl}, 1 \mathrm{mM}$ PMSF, and a proteinase inhibitor cocktail from Roche). The cells were lysed with a Precellys 24 homogenizer at $4^{\circ} \mathrm{C}$, mixed with an equal volume $(600 \mu \mathrm{l})$ of $0.5 \% \mathrm{NP}-40$ in lysis buffer, and incubated at $4^{\circ} \mathrm{C}$ for $15 \mathrm{~min} .50 \mu \mathrm{l}$ of each 
sample was saved (whole-cell lysate), and the remainder was incubated with $100 \mu \mathrm{l}$ of magnetic anti-HA beads (Pierce; 88837) for $1 \mathrm{~h}$ at $4^{\circ} \mathrm{C}$. The beads were washed five times with lysis buffer and incubated at $4^{\circ} \mathrm{C}$ for $5 \mathrm{~min}$ between washes, and the bound proteins were eluted with $40 \mu \mathrm{l}$ SDS-PAGE loading buffer for $15 \mathrm{~min}$ at $50^{\circ} \mathrm{C} .10 \mu \mathrm{l}$ of whole-cell lysate and $20 \mu \mathrm{l}$ of immunoprecipitated proteins were separated by SDS-PAGE and immunoblotted using anti-HA antibody (C29F4; Cell Signaling Technology; \#3724) and anti-GFP (D5.1; Cell Signaling Technology; \#2956).

\section{Assessment of CD55 on the surface of HEK-293 cells by FACS}

Lentiviruses were produced from pLKO.1 expressing shRNA against GPAAl (Mission Sigma-Aldrich; TRCN0000242570), KIAA1109 (Mission Sigma-Aldrich; \#1 TRCNO000282583 and \#2 TRCN0000263340), or negative control (Mission Sigma-Aldrich; nontargeted shRNA control) using standard methods.

HEK-293 cells were grown in DMEM in six-well dishes containing $20 \mu \mathrm{l}$ of lentivirus shRNA and $1.6 \mu \mathrm{l}$ polybrene (Sigma-Aldrich). The medium was removed after $24 \mathrm{~h}$, fresh medium containing $2 \mu \mathrm{g} / \mathrm{ml}$ puromycin (Sigma-Aldrich) was added, and the cultures were grown for $10 \mathrm{~d}$.

The abundance of CD55 on the cell surface was determined as described (Knaus et al., 2018; Tanigawa et al., 2017). Briefly, 100,000 cells were washed two times with the DMEM with $1 \%$ FBS and incubated with $4 \mu \mathrm{l}$ mouse anti-human CD55 conjugated to R-Phycoerythrin (BD Bioscience; 555694) and $16 \mu \mathrm{D}$ DMEM with $1 \% \mathrm{FBS}$ at $25^{\circ} \mathrm{C}$. The cells were washed three times with growth medium, and the fluorescence intensity of 10,000 live cells was measured by FACS (ACSymphony Cell Analyzer; BD Bioscience). Results were analyzed with FlowJo 10.6.0 (BD Bioscience).

\section{qRT-PCR}

Total RNA was extracted from cells (Pure Link RNA kit; Thermo Fisher Scientific), $1 \mu \mathrm{g}$ of total RNA was used to produce cDNA (qScript cDNA SuperMix; Quantabio), and qRT-PCR was performed with the SYBR Green PCR Master Mix (Thermo Fisher Scientific) run on a CFX96 real-time thermal cycler CFX-96 (Bio$\mathrm{Rad})$. The following primers were used: for GAPDH p368 5' -GTC TCCTCTGACTTCAACAGCG-3' and p369 5'-ACCACCCTGTTG CTGTAGCCAA-3'; for GPAA1 p366 5'-TCAATAGCTTCCGCCAGT AC-3' and p367 5'-CCAAGGACCAGGAGCAAGAA-3'; and for KIAA1109/TWEEK p356 5'-CACTCCTGCTATTAAGGGAC-3' and p357 5' -CTCTAATTCACACTGCCATG-3'. All reactions were done in quadruplicate. Relative expression was calculated from the $\mathrm{Cq}$ obtained with CFX manager 3.0 software (Bio-Rad) and with the comparative cycle threshold method, using values from the house keeping gene GAPDH and negative control shRNA.

\section{CRISPR used to generate C. elegans strain AG645}

The Bristol N2 strain was used as the WT for CRISPR/Cas9 genome editing. 20 nucleotide sequences of the lpd-3-specific CRISPR RNAs (crRNAs) were selected using a crRNA design tool (https://www.idtdna.com/site/order/designtool/index/CRISPR_ CUSTOM). All crRNAs and trans-activating crRNA were synthesized by Horizon Discovery. The single-stranded donor oligonucleotides of the lpd-3 repair template were crRNA N-terminus: 5' -GCGAAAACGAAAAAAAATGT-3', PAM site: CGG, crRNA C-terminus: 5' -TTATCCATAATATTGACATC-3', and PAM site: TGG and repair template: $5^{\prime}$-ACGGGTTCTAGG CGGCGGCGAAAACGAAAAAAAATGTGACTAGATGATGGATAA CGACGGAAAAAGGCTTCTATTGGAATA-3'. The oligonucleotides used for genotyping are N-del F1: 5 '-AGTCGGCTACTACGG GTTCT-3', N-del R1: 5'-GATCCAGAAATACCGTACCTCA-3', and inner R1: $5^{\prime}$-CGTTTCTCAGTGGCCGGAAA-3'. Day 1 gravid hermaphrodites $(20)$ were injected with mixed CRISPR/Cas9 reagents as described (Iyer et al., 2019). lpd-3(av254) was generated by CRISPR/Cas 9 mixes that contained two crRNAs at flanking regions of the deleted lpd-3 coding region. Heterozygous lpd-3 N- $\Delta$ animals were screened by PCR (Primer N-del gt F1 and Primer N-del gt R1). The PCR amplicon for the deletion is $403 \mathrm{bp}$. Homozygous mutants were confirmed in subsequent generations.

\section{FLAER staining and microscopy of $C$. elegans}

Staining with FLAER (Proaerolysin conjugated Alexa Fluor 488; Cedarlane Corporation; FL1S-C) was adapted from previous studies (Budirahardja et al., 2015; Murata et al., 2012). 23-gauge needles were used to dissect the embryos from the gravid hermaphrodites in M9 buffer, and the embryos were covered with an $18 \times 18-\mathrm{mm}$ coverslip. The embryos were then freeze-cracked and fixed in methanol at $-20^{\circ} \mathrm{C}$ for $10 \mathrm{~min}$. The slides were washed twice in PBS for $5 \mathrm{~min}$ and blocked with $2 \%$ bovine serum albumin solution for $30 \mathrm{~min}$ at room temperature. The slides were incubated in $50 \mathrm{nM}$ FLAER staining solution for $2 \mathrm{~h}$. After incubation, the slides were washed twice with PBS for 5 min and mounted in VECTASHIELD (Vector Labs) mounting medium containing $5 \mu \mathrm{g} / \mathrm{ml}$ DAPI overnight before imaging.

FLAER stained slides were imaged by a spinning disk confocal system that includes a Photometrics Prime 95B EMCCD camera and a Yokogawa CSU-X1 confocal scanner unit. Images were acquired by Nikon's NIS imaging software using a Nikon $60 \times 1.2$ NA water objective with $0.5-\mu \mathrm{m}$ z-step size; $30-50$ planes were captured. FLAER signals were quantified using ImageJ/FIJI Bioformat plugin (Linkert et al., 2010; Schindelin et al., 2012). Both the Shapiro-Wilk and Kolmogorov-Smirnov normality tests indicated that all data follow normal distributions.

For differential interference contrast (DIC) imaging of lpd-3 $N-\Delta$ mutants, animals were immobilized on $7 \%$ agar pads with anesthetic (0.01\% levamisole in M9 buffer). DIC image acquisition was performed using a Nikon $60 \times 1.2$ NA water objective with a 1- $\mu \mathrm{m}$ z-step size; 15-20 planes were captured.

\section{Online supplemental material}

Fig. S1 presents material that supports the findings shown in Fig. 1. Fig. S2 presents material that supports the findings shown in Fig. 2, Fig. 3, and Fig. 4. Fig. S3 presents material that supports the findings shown in Fig. 5. Video 1 shows the structure of S. cerevisiae Vps13 generated with RoseTTAFold. Video 2 shows the structure of $S$. cerevisiae Atg2 generated with RoseTTAFold. Video 3 shows the structure of $S$. cerevisiae Csfl generated with RoseTTAFold. Video 4 shows the structure of S. cerevisiae Fmp27 generated with RoseTTAFold. Video 5 shows the structure of 
S. cerevisiae Hob2 generated with RoseTTAFold. Table S1 lists the $S$. cerevisiae strains used in this study.

\section{Acknowledgments}

We thank A. Ferguson for technical assistance, the Caenorhabditis Genetics Center (National Institutes of Health, Office of Research Infrastructure Programs, P40OD010440) for C. elegans strains, the Flow Cytometry Core of the National Heart, Lung, and Blood Institute and D. Masison (National Institute of Diabetes and Digestive and Kidney Diseases, Bethesda, MD) for reagents, J. Nunnari (University of California, Davis, Davis, CA) and O. Cohen-Fix (National Institute of Diabetes and Digestive and Kidney Diseases, Bethesda, MD) for plasmids, Y. Murakami and T. Kinoshita (Osaka University, Osaka, Japan) for cell lines and helpful advice, and T. Balla for reading the manuscript.

This research was supported by the Intramural Research Program of the National Institutes of Health, National Institute of Diabetes and Digestive and Kidney Diseases.

The authors declare no completing financial interests.

Author contributions: A. Toulmay conceptualized the project, supervised members of the Prinz laboratory, and prepared figures. F.B. Whittle, J. Yang, X. Bai, J. Diarra, and S. Banerjee conducted the investigation. T.P. Levine and A. Golden conceptualized the project. W.A. Prinz wrote the manuscript.

Submitted: 19 November 2021

Revised: 14 December 2021

Accepted: 15 December 2021

\section{References}

Acoba, M.G., N. Senoo, and S.M. Claypool. 2020. Phospholipid ebb and flow makes mitochondria go. J. Cell Biol. 219:e202003131. https://doi.org/10 $.1083 /$ jcb.202003131

Benghezal, M., P.N. Lipke, and A. Conzelmann. 1995. Identification of six complementation classes involved in the biosynthesis of glycosylphosphatidylinositol anchors in Saccharomyces cerevisiae. J. Cell Biol. 130:1333-1344. https://doi.org/10.1083/jcb.130.6.1333

Budirahardja, Y., T.D. Doan, and R. Zaidel-Bar. 2015. Glycosyl phosphatidylinositol anchor biosynthesis is essential for maintaining epithelial integrity during Caenorhabditis elegans embryogenesis. PLoS Genet. 11: e1005082. https://doi.org/10.1371/journal.pgen.1005082

Calzada, E., O. Onguka, and S.M. Claypool. 2016. Phosphatidylethanolamine Metabolism in Health and Disease. Int. Rev. Cell Mol. Biol. 321:29-88. https://doi.org/10.1016/bs.ircmb.2015.10.001

Chiapparino, A., K. Maeda, D. Turei, J. Saez-Rodriguez, and A.C. Gavin. 2016. The orchestra of lipid-transfer proteins at the crossroads between metabolism and signaling. Prog. Lipid Res. 61:30-39. https://doi.org/10 $.1016 /$ j.plipres.2015.10.004

Chowdhury, S., C. Otomo, A. Leitner, K. Ohashi, R. Aebersold, G.C. Lander, and T. Otomo. 2018. Insights into autophagosome biogenesis from structural and biochemical analyses of the ATG2A-WIPI4 complex. Proc. Natl. Acad. Sci. USA. 115:E9792-E9801. https://doi.org/10.1073/pnas .1811874115

Copic, A., M. Dorrington, S. Pagant, J. Barry, M.C. Lee, I. Singh, J.L. Hartman IV, and E.A. Miller. 2009. Genomewide analysis reveals novel pathways affecting endoplasmic reticulum homeostasis, protein modification and quality control. Genetics. 182:757-769. https://doi.org/10.1534/genetics .109 .101105

De, M., A.N. Oleskie, M. Ayyash, S. Dutta, L. Mancour, M.E. Abazeed, E.J. Brace, G. Skiniotis, and R.S. Fuller. 2017. The Vps13p-Cdc31p complex is directly required for TGN late endosome transport and TGN homotypic fusion. J. Cell Biol. 216:425-439. https://doi.org/10.1083/jcb.201606078
Flury, I., A. Benachour, and A. Conzelmann. 2000. YLL031c belongs to a novel family of membrane proteins involved in the transfer of ethanolaminephosphate onto the core structure of glycosylphosphatidylinositol anchors in yeast. J. Biol. Chem. 275:24458-24465. https://doi.org/10 $.1074 / \mathrm{jbc} . \mathrm{M003844200}$

Friedman, J.R., M. Kannan, A. Toulmay, C.H. Jan, J.S. Weissman, W.A. Prinz, and J. Nunnari. 2018. Lipid Homeostasis Is Maintained by Dual Targeting of the Mitochondrial PE Biosynthesis Enzyme to the ER. Dev. Cell. 44:261-270.e6. https://doi.org/10.1016/j.devcel.2017.11.023

Fujita, M., and T. Kinoshita. 2012. GPI-anchor remodeling: potential functions of GPI-anchors in intracellular trafficking and membrane dynamics. Biochim. Biophys. Acta. 1821:1050-1058. https://doi.org/10.1016/j.bbalip .2012.01.004

Gauthier, J., I.A. Meijer, D. Lessel, N.E. Mencacci, D. Krainc, M. Hempel, K. Tsiakas, H. Prokisch, E. Rossignol, M.H. Helm, et al. 2018. Recessive mutations in VPS13D cause childhood onset movement disorders. Ann. Neurol. 83:1089-1095. https://doi.org/10.1002/ana.25204

Gueneau, L., R.J. Fish, H.E. Shamseldin, N. Voisin, F. Tran Mau-Them, E. Preiksaitiene, G.R. Monroe, A. Lai, A. Putoux, F. Allias, et al. DDD Study. 2018. KIAA1109 Variants Are Associated with a Severe Disorder of Brain Development and Arthrogryposis. Am. J. Hum. Genet. 102:116-132. https://doi.org/10.1016/j.ajhg.2017.12.002

Hiroi, Y., I. Komuro, R. Chen, T. Hosoda, T. Mizuno, S. Kudoh, S.P. Georgescu, M.E. Medof, and Y. Yazaki. 1998. Molecular cloning of human homolog of yeast GAAl which is required for attachment of glycosylphosphatidylinositols to proteins. FEBS Lett. 421:252-258. https:// doi.org/10.1016/S0014-5793(97)01576-7

Ichimura, Y., T. Kirisako, T. Takao, Y. Satomi, Y. Shimonishi, N. Ishihara, N. Mizushima, I. Tanida, E. Kominami, M. Ohsumi, et al. 2000. A ubiquitin-like system mediates protein lipidation. Nature. 408:488-492. https://doi.org/10.1038/35044114

Iyer, J., N. DeVaul, T. Hansen, and B. Nebenfuehr. 2019. Using Microinjection to Generate Genetically Modified Caenorhabditis elegans by CRISPR/ Cas9 Editing. Methods Mol. Biol. 1874:431-457. https://doi.org/10.1007/ 978-1-4939-8831-0_25

John Peter, A.T., S.N.S. van Schie, N.J. Cheung, A.H. Michel, M. Peter, and B. Kornmann. 2021. Rewiring phospholipid biosynthesis reveals robustness in membrane homeostasis and uncovers lipid regulatory players. bioRxiv. doi:10.1101/2021.07.21.453065 (Preprint posted July 21, 2021)

Kannan, M., S. Lahiri, L.K. Liu, V. Choudhary, and W.A. Prinz. 2017. Phosphatidylserine synthesis at membrane contact sites promotes its transport out of the ER. J. Lipid Res. 58:553-562. https://doi.org/10.1194/ jlr.M072959

Kawano, S., Y. Tamura, R. Kojima, S. Bala, E. Asai, A.H. Michel, B. Kornmann, I. Riezman, H. Riezman, Y. Sakae, et al. 2018. Structure-function insights into direct lipid transfer between membranes by $\mathrm{Mmm1}$ Mdm12 of ERMES. J. Cell Biol. 217:959-974. https://doi.org/10.1083/ jcb.201704119

Knaus, A., J.T. Pantel, M. Pendziwiat, N. Hajjir, M. Zhao, T.C. Hsieh, M. Schubach, Y. Gurovich, N. Fleischer, M. Jäger, et al. 2018. Characterization of glycosylphosphatidylinositol biosynthesis defects by clinical features, flow cytometry, and automated image analysis. Genome Med. 10:3. https://doi.org/10.1186/s13073-017-0510-5

Kolehmainen, J., G.C. Black, A. Saarinen, K. Chandler, J. Clayton-Smith, A.L. Träskelin, R. Perveen, S. Kivitie-Kallio, R. Norio, M. Warburg, et al 2003. Cohen syndrome is caused by mutations in a novel gene, COH1, encoding a transmembrane protein with a presumed role in vesiclemediated sorting and intracellular protein transport. Am. J. Hum. Genet. 72:1359-1369. https://doi.org/10.1086/375454

Kornmann, B., E. Currie, S.R. Collins, M. Schuldiner, J. Nunnari, J.S. Weissman, and P. Walter. 2009. An ER-mitochondria tethering complex revealed by a synthetic biology screen. Science. 325:477-481. https://doi .org/10.1126/science. 1175088

Kumar, N., M. Leonzino, W. Hancock-Cerutti, F.A. Horenkamp, P. Li, J.A Lees, H. Wheeler, K.M. Reinisch, and P. De Camilli. 2018. VPS13A and VPS13C are lipid transport proteins differentially localized at ER contact sites. J. Cell Biol. 217:3625-3639. https://doi.org/10.1083/jcb.201807019

Lang, A.B., A.T. John Peter, P. Walter, and B. Kornmann. 2015. ERmitochondrial junctions can be bypassed by dominant mutations in the endosomal protein Vps13. J. Cell Biol. 210:883-890. https://doi.org/ $10.1083 /$ jcb. 201502105

Leonzino, M., K.M. Reinisch, and P. De Camilli. 2021. Insights into VPS13 properties and function reveal a new mechanism of eukaryotic lipid transport. Biochim. Biophys. Acta Mol. Cell Biol. Lipids. 1866:159003. https://doi.org/10.1016/j.bbalip.2021.159003 
Lesage, S., V. Drouet, E. Majounie, V. Deramecourt, M. Jacoupy, A. Nicolas, F. Cormier-Dequaire, S.M. Hassoun, C. Pujol, S. Ciura, et al. International Parkinson's Disease Genomics Consortium (IPDGC). 2016. Loss of VPS13C Function in Autosomal-Recessive Parkinsonism Causes Mitochondrial Dysfunction and Increases PINK1/Parkin-Dependent Mitophagy. Am. J. Hum. Genet. 98:500-513. https://doi.org/10.1016/j.ajhg .2016.01.014

Li, P., J.A. Lees, C.P. Lusk, and K.M. Reinisch. 2020. Cryo-EM reconstruction of a VPS13 fragment reveals a long groove to channel lipids between membranes. J. Cell Biol. 219:e202001161. https://doi.org/10.1083/jcb .202001161

Linkert, M., C.T. Rueden, C. Allan, J.M. Burel, W. Moore, A. Patterson, B. Loranger, J. Moore, C. Neves, D. Macdonald, et al. 2010. Metadata matters: access to image data in the real world. J. Cell Biol. 189:777-782. https://doi.org/10.1083/jcb.201004104

Maeda, S., C. Otomo, and T. Otomo. 2019. The autophagic membrane tether ATG2A transfers lipids between membranes. eLife. 8:e45777. https://doi .org/10.7554/eLife.45777

Makhija, S., D. Brown, R.M. Rudlaff, J.K. Doh, S. Bourke, Y. Wang, S. Zhou, R. Cheloor-Kovilakam, and B. Huang. 2021. Versatile Labeling and Detection of Endogenous Proteins Using Tag-Assisted Split Enzyme Complementation. ACS Chem. Biol. 16:671-681. https://doi.org/10.1021/ acschembio.0c00925

Menon, A.K., and V.L. Stevens. 1992. Phosphatidylethanolamine is the donor of the ethanolamine residue linking a glycosylphosphatidylinositol anchor to protein. J. Biol. Chem. 267:15277-15280. https://doi.org/10 .1016/S0021-9258(19)49529-X

Murata, D., K.H. Nomura, K. Dejima, S. Mizuguchi, N. Kawasaki, Y. Matsuishi-Nakajima, S. Ito, K. Gengyo-Ando, E. Kage-Nakadai, S. Mitani, and K. Nomura. 2012. GPI-anchor synthesis is indispensable for the germline development of the nematode Caenorhabditis elegans. Mol. Biol. Cell. 23:982-995. https://doi.org/10.1091/mbc.e10-10-0855

Neuman, S.D., J.R. Jorgensen, A.T. Cavanagh, J.T. Smyth, J.E. Selegue, S.D. Emr, and A. Bashirullah. 2022. The Hob proteins are novel and conserved lipid-binding proteins at ER-PM contact sites. J. Cell Sci. 135: jcs.259086. https://doi.org/10.1242/jcs.259086

Park, J.S., M.K. Thorsness, R. Policastro, L.L. McGoldrick, N.M. Hollingsworth, P.E. Thorsness, and A.M. Neiman. 2016. Yeast Vps13 promotes mitochondrial function and is localized at membrane contact sites. Mol. Biol. Cell. 27:2435-2449. https://doi.org/10.1091/mbc.e16-02-0112

Patel, D., and S.N. Witt. 2017. Ethanolamine and Phosphatidylethanolamine: Partners in Health and Disease. Oxid. Med. Cell. Longev. 2017:4829180. https://doi.org/10.1155/2017/4829180

Petrungaro, C., and B. Kornmann. 2019. Lipid exchange at ER-mitochondria contact sites: a puzzle falling into place with quite a few pieces missing. Curr. Opin. Cell Biol. 57:71-76. https://doi.org/10.1016/j.ceb.2018.11.005

Rampoldi, L., C. Dobson-Stone, J.P. Rubio, A. Danek, R.M. Chalmers, N.W. Wood, C. Verellen, X. Ferrer, A. Malandrini, G.M. Fabrizi, et al. 2001. A conserved sorting-associated protein is mutant in choreaacanthocytosis. Nat. Genet. 28:119-120. https://doi.org/10.1038/88821

Raychaudhuri, S., and W.A. Prinz. 2008. Nonvesicular phospholipid transfer between peroxisomes and the endoplasmic reticulum. Proc. Natl. Acad. Sci. USA. 105:15785-15790. https://doi.org/10.1073/pnas.0808321105

Reggiori, F., E. Canivenc-Gansel, and A. Conzelmann. 1997. Lipid remodeling leads to the introduction and exchange of defined ceramides on GPI proteins in the ER and Golgi of Saccharomyces cerevisiae. EMBO J. 16: 3506-3518. https://doi.org/10.1093/emboj/16.12.3506

Reinisch, K.M., and W.A. Prinz. 2021. Mechanisms of nonvesicular lipid transport. J. Cell Biol. 220:e202012058. https://doi.org/10.1083/jcb .202012058

Schindelin, J., I. Arganda-Carreras, E. Frise, V. Kaynig, M. Longair, T. Pietzsch, S. Preibisch, C. Rueden, S. Saalfeld, B. Schmid, et al. 2012. Fiji: an open-source platform for biological-image analysis. Nat. Methods. 9: 676-682. https://doi.org/10.1038/nmeth.2019

Seong, E., R. Insolera, M. Dulovic, E.J. Kamsteeg, J. Trinh, N. Brüggemann, E. Sandford, S. Li, A.B. Ozel, J.Z. Li, et al. 2018. Mutations in VPS13D lead to a new recessive ataxia with spasticity and mitochondrial defects. Ann. Neurol. 83:1075-1088. https://doi.org/10.1002/ana.25220

Shintani, T., and D.J. Klionsky. 2004. Cargo proteins facilitate the formation of transport vesicles in the cytoplasm to vacuole targeting pathway. J. Biol. Chem. 279:29889-29894. https://doi.org/10.1074/jbc .M404399200

Suzuki, K., M. Akioka, C. Kondo-Kakuta, H. Yamamoto, and Y. Ohsumi. 2013 Fine mapping of autophagy-related proteins during autophagosome formation in Saccharomyces cerevisiae. J. Cell Sci. 126:2534-2544. https://doi.org/10.1242/jcs.122960

Tanigawa, J., H. Mimatsu, S. Mizuno, N. Okamoto, D. Fukushi, K. Tominaga H. Kidokoro, Y. Muramatsu, E. Nishi, S. Nakamura, et al. 2017. Phenotype-genotype correlations of PIGO deficiency with variable phenotypes from infantile lethality to mild learning difficulties. Hum Mutat. 38:805-815. https://doi.org/10.1002/humu.23219

Tatsuta, T., and T. Langer. 2017. Intramitochondrial phospholipid trafficking. Biochim. Biophys. Acta Mol. Cell Biol. Lipids. 1862:81-89. https://doi.org/ 10.1016/j.bbalip.2016.08.006

Tokai, M., H. Kawasaki, Y. Kikuchi, and K. Ouchi. 2000. Cloning and characterization of the CSF1 gene of Saccharomyces cerevisiae, which is required for nutrient uptake at low temperature. J. Bacteriol. 182: 2865-2868. https://doi.org/10.1128/JB.182.10.2865-2868.2000

Toulmay, A., and W.A. Prinz. 2012. A conserved membrane-binding domain targets proteins to organelle contact sites. J. Cell Sci. 125:49-58. https:// doi.org/10.1242/jcs.085118

Tunyasuvunakool, K., J. Adler, Z. Wu, T. Green, M. Zielinski, A. Žídek, A. Bridgland, A. Cowie, C. Meyer, A. Laydon, et al. 2021. Highly accurate protein structure prediction for the human proteome. Nature. 596: 590-596. https://doi.org/10.1038/s41586-021-03828-1

Valverde, D.P., S. Yu, V. Boggavarapu, N. Kumar, J.A. Lees, T. Walz, K.M. Reinisch, and T.J. Melia. 2019. ATG2 transports lipids to promote autophagosome biogenesis. J. Cell Biol. 218:1787-1798. https://doi.org/10 $.1083 /$ jcb.201811139

Vidugiriene, J., D.K. Sharma, T.K. Smith, N.A. Baumann, and A.K. Menon 1999. Segregation of glycosylphosphatidylinositol biosynthetic reactions in a subcompartment of the endoplasmic reticulum. J. Biol. Chem. 274:15203-15212. https://doi.org/10.1074/jbc.274.21.15203

Wong, L.H., A.T. Gatta, and T.P. Levine. 2019. Lipid transfer proteins: the lipid commute via shuttles, bridges and tubes. Nat. Rev. Mol. Cell Biol. 20:85-101. https://doi.org/10.1038/s41580-018-0071-5

Yang, J., I. Anishchenko, H. Park, Z. Peng, S. Ovchinnikov, and D. Baker. 2020. Improved protein structure prediction using predicted interresidue orientations. Proc. Natl. Acad. Sci. USA. 117:1496-1503. https:// doi.org/10.1073/pnas.1914677117 


\section{Supplemental material}

A

\begin{tabular}{|c|c|c|} 
P-values & $1^{\text {st }}$ Structure from AlphaFold & $\begin{array}{c}1^{\text {st }} \text { structure from } \\
\text { RoseTTAFold }\end{array}$ \\
\hline Atg2 & $1.24 \mathrm{E}-09$ & $1.27 \mathrm{E}-09$ \\
\hline Fmp27 & $3.40 \mathrm{E}-07$ & $3.31 \mathrm{E}-07$ \\
\hline Hob2 & $3.96 \mathrm{E}-11$ & $3.97 \mathrm{E}-11$ \\
\hline
\end{tabular}

B
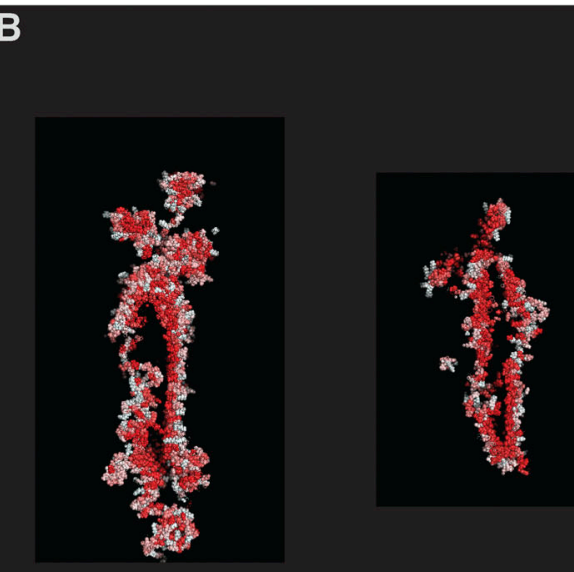

Atg2

Csf1

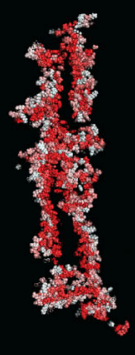

Fmp27

Hob2

Figure S1. Similarity of Vps13 homologues. (A) Significance of the structural similarity between pairs of proteins as determined by FATCAT. P values are from pairwise comparisons of the structures of the indicated proteins as determined by RoseTTAFold and AlphaFold. (B) Space-filling models of the five Vps13like proteins in S. cerevisiae were generated using PyMol from the predicted structures obtained from RoseTTAFold. Longitudinal cross sections are shown with hydrophobic residues colored red. All five proteins have a central hydrophobic groove. 
A

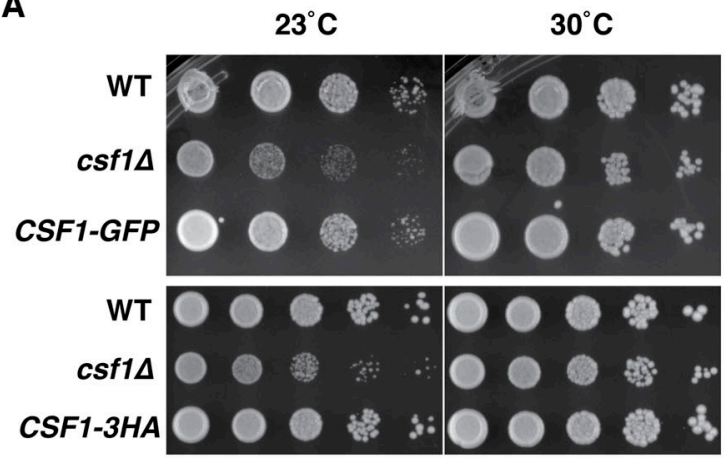

C

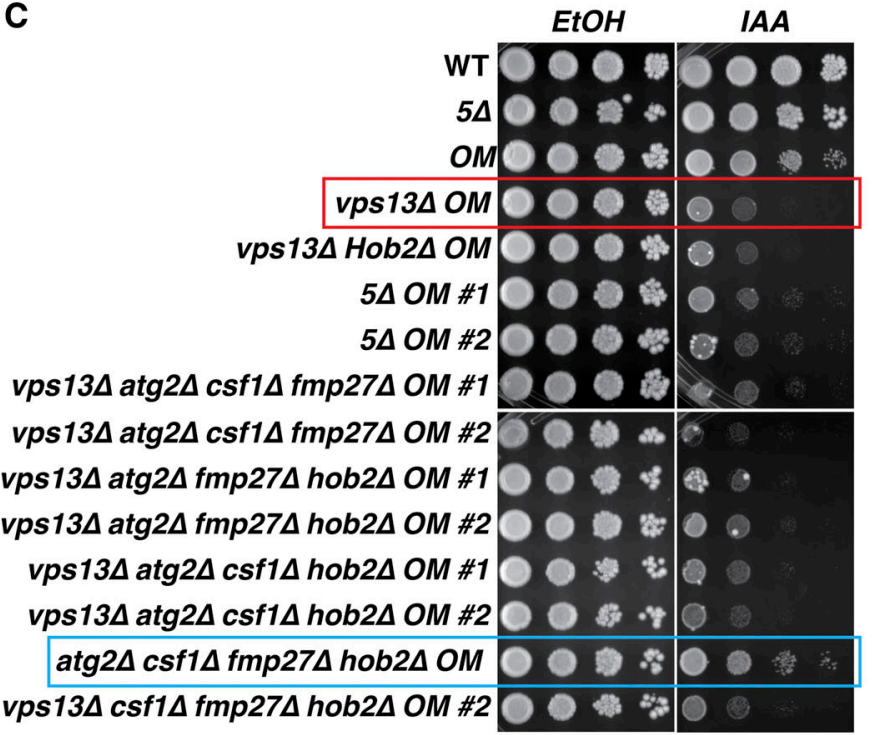

B
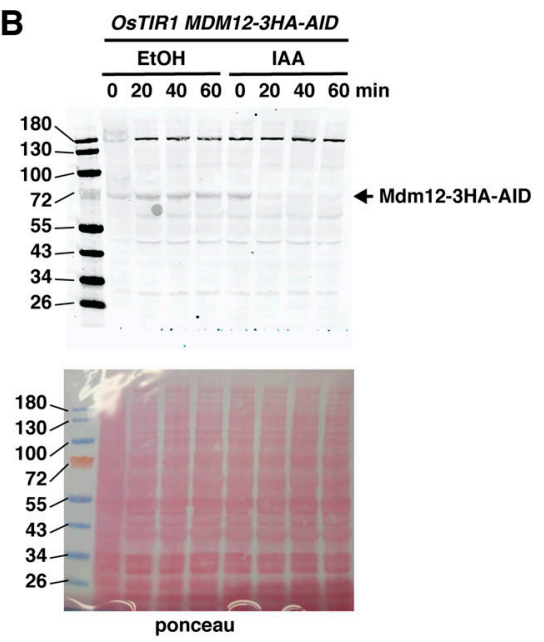

D

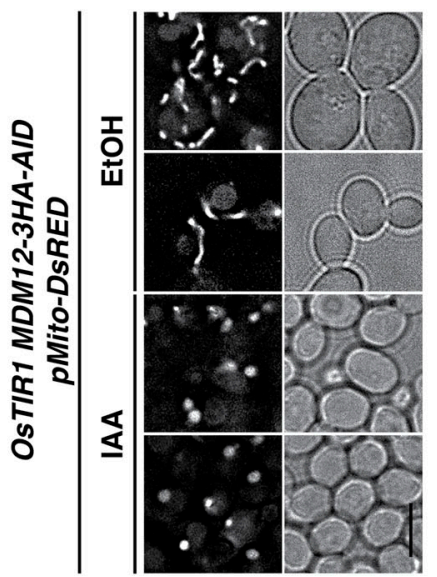

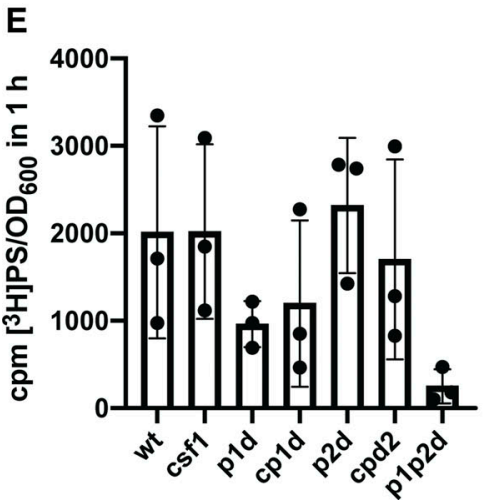

F

$\mathrm{t}=\mathbf{3 h}$

$55 \mathrm{kD}$

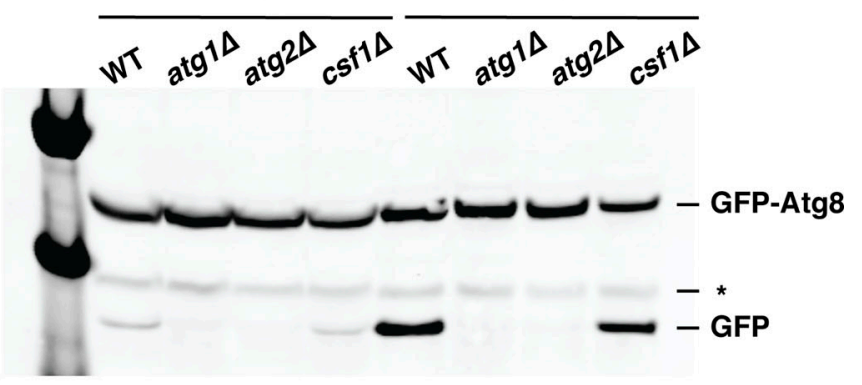

Figure S2. Csf1 does not play a role in autophagy and other controls for findings in Fig. 2, Fig. 3, and Fig. 4. (A) 10-fold serial dilutions of the indicated strains grown on SC plates. The plates were grown for $3 \mathrm{~d}$. (B) WT cells expressing Oryza sativa TIR1 (OsTIR1) and Mdm12 with a C-terminal fusion to $3 \mathrm{HA}$ antigen tags and the auxin-inducible degron (Mdm12-3HA-AID) were grown in SC medium and the auxin IAA or ethanol (EtOH) were added. Samples were taken at the indicated times, and proteins were extracted. The proteins were separated by SDS-PAGE and transferred to a nitrocellulose membrane. The membrane was stained with Ponceau (bottom) and immunoblotted with anti-HA antibodies (top). (C) 10 -fold serial dilutions of the indicated strains grown for $3 \mathrm{~d}$ on YPD plates containing IAA or ethanol (EtOH). OM, strains expressing OsTIR1 and Mdm12-3HA-AID (described in B). Numbers indicate size of molecular weight markers $(\mathrm{kDa}$ ). Note that IAA prevents the growth of vps $13 \triangle \mathrm{OM}$ cells (red box) but not atg $2 \Delta$ csf $1 \triangle$ fmp27 hob2 $0 M$ cells (blue box), indicating that Vps13 is the only one of the five Vps13-like proteins required for viability of cells lacking ERMES. (D) Cells expressing OsTIR1 and Mdm12-3HA-AID (described in $\mathrm{B}$ ) and the mitochondrial marker Mito-DsRed (expressed from the plasmid pMito-DsRed) were grown in SC medium and visualized live $1 \mathrm{~h}$ after addition of IAA or ethanol $(\mathrm{EtOH})$ to the medium. Bar $=5 \mu \mathrm{m}$. (E) Total $\left[{ }^{3} \mathrm{H}\right] \mathrm{PS}$ formed $\left(\left[{ }^{3} \mathrm{H}\right] \mathrm{PS}+\left[{ }^{3} \mathrm{H}\right] \mathrm{PE}+\left[{ }^{3} \mathrm{H}\right] \mathrm{PC}\right)$ during the 1-h labeling with $\left[{ }^{3} \mathrm{H}\right]$ serine shown in Fig. $3 \mathrm{~F}$ (mean \pm SD from three independent experiments). (F) Strains with the indicated genotypes expressing GFP-Atg8 were grown in SC medium to logarithmic growth phase and transferred to SD-N medium (synthetic dextrose, lacking amino acid supplementation). Samples were taken at the indicated times and immunoblotted with an anti-GFP antibody. ${ }^{*}$, a nonspecific protein bound by the anti-GFP antibody. Source data are available for this figure: SourceData FS2. 
A

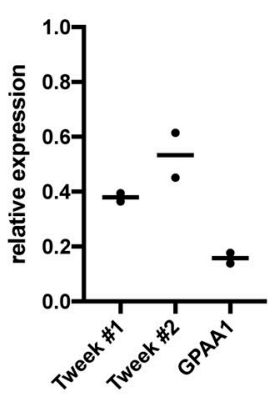

C

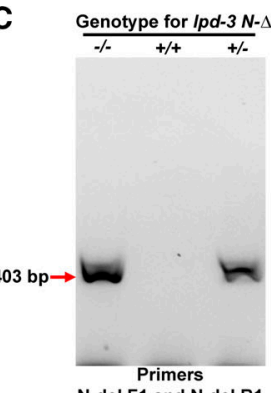

N-del F1 and N-del R1

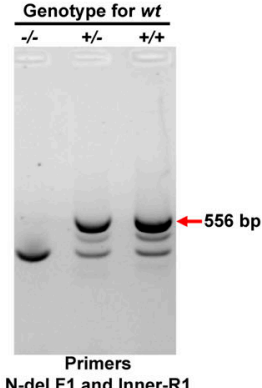

N-del F1 and Inner-R1

B

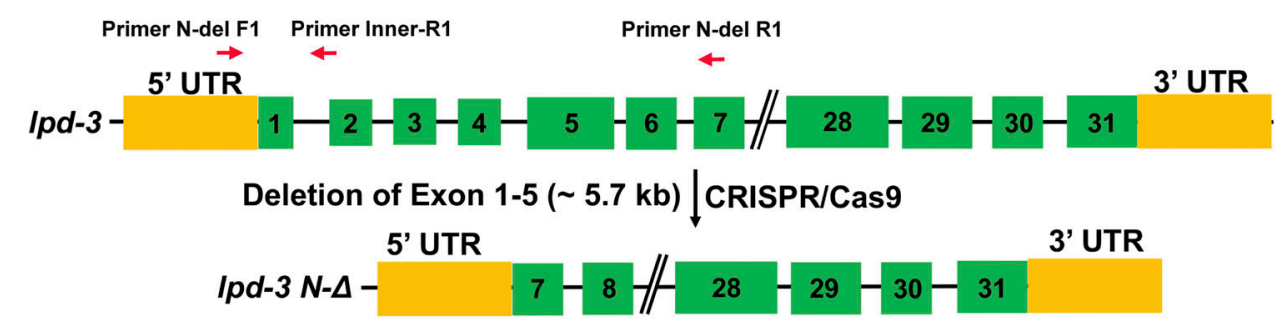

D

Ipd-35'UTR

Stop-in Cassette

Repair Template acgggttctaggcggcggcgaaaacgaaaaaaATGTGACtAgATgATGGATAACGACGGAAAAGGCTTCTATTGGAATA ||||||||||||||||||||||||||||||||||l|l|l|l|l|T|||||||||||||||||||||||||||||||||| Ipd-3 $\mathrm{N}-\Delta$ Sequencing ACGGGTTCTAGGCGGCGGCGAAACGAAAAAAAATGTGACTAGATGATGGATAACGACGGAAAAAGGCTTCTATTGGAATA

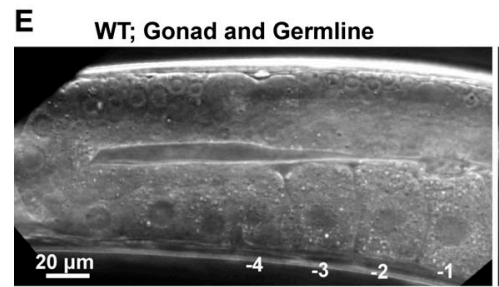

$\mathbf{F}$ WT; FLAER; 4-Cell Stage

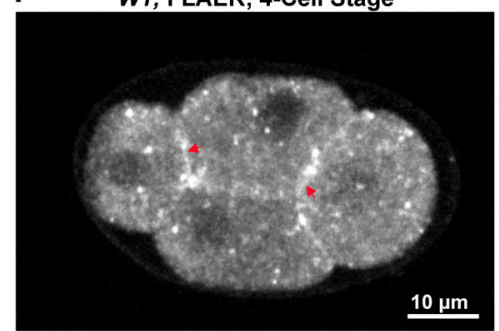

Ipd-3 N- $\Delta$; FLAER; 4-Cell Stage

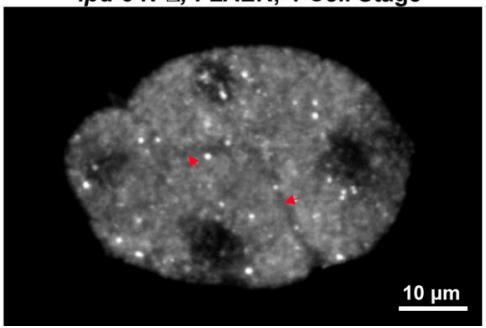

Ipd-3 N- $\Delta$; Gonad and Germline

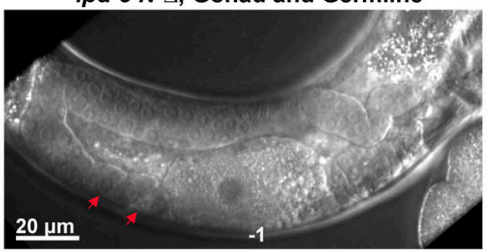

WT; DAPI; 4-Cell Stage

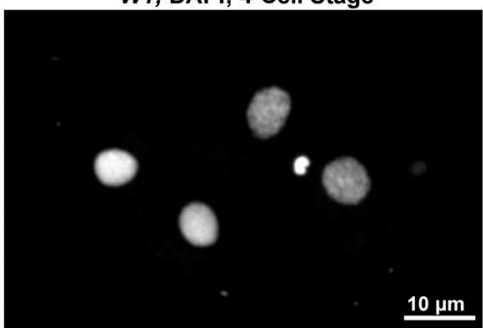

Ipd-3 N- $\Delta$; DAPI; 4-Cell Stage

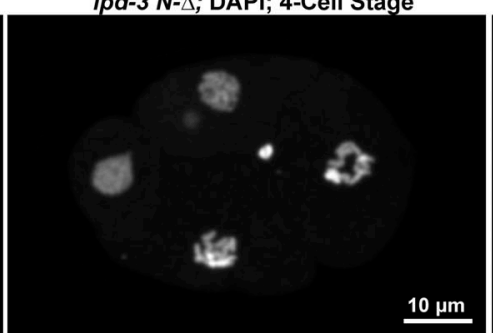

Ipd-3 N- $\Delta$; Gonad and Germline

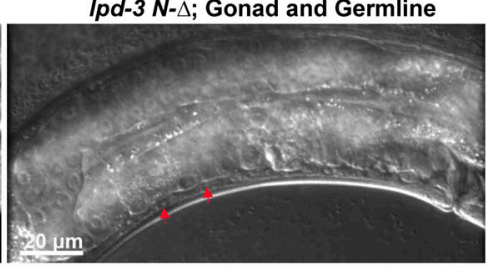

MERGE

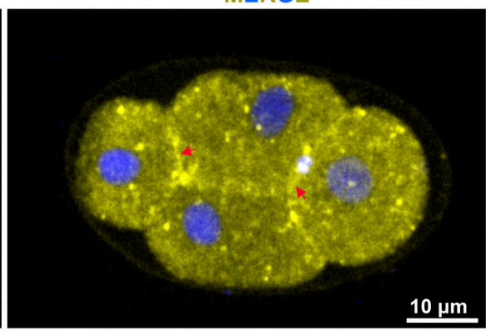

MERGE

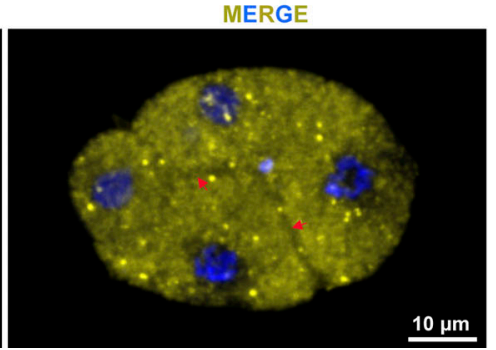

Figure S3. Supporting information for the results shown in Fig. 5. (A) Relative expression of the GPAA1 and TWEEK genes for the experiments shown in Fig. $5 \mathrm{~A}$ as determined by qRT-PCR. The mean of two independent experiments ( $n=4$ per experiment). (B) Position of the $\mathrm{C}$. elegans genotyping primers ( $\mathrm{N}$-del F1, N-del R1, and Inner R1) for verifying the knockout allele (red arrows); exons in green and UTRs in yellow. (C) Representative PCR results from genotyping single animals for lpd-3 N- $\Delta$ candidates. Two primers (two flanking primers N-del F1 and N-del R1 located outside the deleted region) were used to genotype for candidate lpd-3(av254) deletion animals. A homozygous deletion (-/-) was indicated by a 403-bp amplicon, which was amplified by N-del F1 and N-del R1. In WT $(+/+)$, the PCR product amplified by N-del F1 and the inner primer Inner-R1 is 556 bp. In heterogenous animals (+/-), both amplicons will be amplified. (D) Homozygous Ipd-3 N- $\Delta$ animals were confirmed by Sanger sequencing, which has a "STOP-IN cassette" (red lines) after start codon. (E) DIC microscopy revealed that deformed gonads and germlines were frequently observed in lpd-3 N- $\Delta$ animals. Bars $=20 \mu \mathrm{m}$. (F) FLAER staining of four-cell-stage $C$. elegans embryos from WT and lpd-3 N- $\Delta$ animals. The nuclei were stained with DAPI. Red arrows indicate cleavage furrows. Bars $=10 \mu m$. Source data are available for this figure: SourceData FS3. 
Video 1. Structure of S. cerevisiae Vps13 generated with RoseTTAFold and ChimeraX: $\beta$-sheets (blue), a-helices (red), and coil (green). The movie shows rotation around the longitudinal axis. Frame rate $=5$ frames per second.

Video 2. Structure of S. cerevisiae Atg2 generated with RoseTTAFold and ChimeraX: $\beta$-sheets (blue), $\boldsymbol{a}$-helices (red), and coil (green). The movie shows rotation around the longitudinal axis. Frame rate $=5$ frames per second.

Video 3. Structure of S. cerevisiae Csf1 generated with RoseTTAFold and ChimeraX: $\beta$-sheets (blue), $a$-helices (red), and coil (green). The movie shows rotation around the longitudinal axis. Frame rate $=5$ frames per second.

Video 4. Structure of S. cerevisiae Fmp27 generated with RoseTTAFold and ChimeraX: $\beta$-sheets (blue), a-helices (red), and coil (green). The movie shows rotation around the longitudinal axis. Frame rate $=5$ frames per second.

Video 5. Structure of S. cerevisiae Hob2 generated with RoseTTAFold and ChimeraX: $\beta$-sheets (blue), a-helices (red), and coil (green). The movie shows rotation around the longitudinal axis. Frame rate $=5$ frames per second.

Provided online is one table. Table S1 lists the S. cerevisiae strains used in this study. 